\title{
A mathematical model demonstrating the role of interstitial fluid flow on the clearance and accumulation of amyloid $\beta$ in the brain
}

\author{
C. Y. Chen*, Y. H. Tseng* and J. P. Ward ${ }^{+}$ \\ * Department of Applied Mathematics, National University of Kaohsiung, \\ Kaohsiung, Taiwan \\ + School of Mathematical Sciences, Loughborough University, \\ Loughborough, U.K.
}

September 12, 2019

\begin{abstract}
A system of partial differential equations is developed to describe the formation and clearance of amyloid $\beta(\mathrm{A} \beta)$ and the subsequent buildup of $\mathrm{A} \beta$ plaques in the brain, which are associated with Alzheimer's disease. The $\mathrm{A} \beta$ related proteins are divided into five distinct categories depending on their size. In addition to enzymatic degradation, the clearance via diffusion and the outflow of interstitial fluid (ISF) into the surrounding cerebral spinal fluid (CSF) are considered. Treating the brain tissue as a porous medium, a simplified two-dimensional circular geometry is assumed for the transverse section of the brain leading to a nonlinear, coupled system of PDEs. Asymptotic analysis is carried out for the steady states of the spatially homogeneous system in the vanishingly small limit of $\mathrm{A} \beta$ clearance rate. The PDE model is studied numerically for two cases, a spherically symmetric case and a more realistic $2 \mathrm{D}$ asymmetric case, allowing for nonuniform boundary conditions. Our investigations demonstrate that ISF advection is a key component in reproducing the clinically observed accumulation of plaques on the outer boundaries. Furthermore, ISF circulation serves to enhance A $\beta$ clearance over diffusion alone and that non-uniformities in ISF drainage into the CSF can lead to local clustering of plaques. Analysis of the model also demonstrates that plaque formation does not directly correspond to the high presence of toxic oligomers.
\end{abstract}

Keywords: mathematical model, Alzheimer's disease, amyloid $\beta$, brain, interstitial fluid, advection, plaque formation.

\section{Introduction}

Alzheimer's disease (AD) is the most common neurodegenerative disease associated with ageing and the incidence increases dramatically with age, affecting 50\% of those over 90 [1]. It is the main cause of dementia, with patients exhibiting progressive memory loss 
and cognitive decline. These symptoms become more severe with the progression of the disease, eventually culminating in the loss of executive functions. As the life expectancy increases, senile dementia is becoming more prevalent. Extensive research has been carried out to study the pathogenesis of the disease, but in spite of much effort and progress, the understanding remains incomplete. At present, no effective treatment is available to halt or alter the progression of the disease except for a limited number of options to help managing the symptoms.

$\mathrm{AD}$ is characterised pathologically by the presence of two lesions: amyloid plaques that consist mainly of the extracellular deposits of amyloid $\beta(\mathrm{A} \beta)$ plaques and intracellular neurofibrillary tangle consisting of twisted filaments of hyper-phosphorylated tau protein $[1,2]$. Although the pathogenesis is complex and still difficult to pinpoint, strong evidence has been put forward supporting the amyloid cascade hypothesis of AD which postulates an early and vital role of $\mathrm{A} \beta$ as a trigger of a cascade of events leading to synaptic dysfunction and eventual neuronal loss [3].

Amyloid $\beta$ is a natural product of the human body and is present in the brain of a healthy person through life. The peptides are eliminated physiologically in three ways: through capillary reabsorption, enzymatic degradation or being transported into the surround cerebral spinal fluid (CSF) for clearance; all three ways are affected in ageing [1]. The mere presence of $\mathrm{A} \beta$ therefore are not expected to cause neurodegeneration; the self-association of $\mathrm{A} \beta$ monomers into larger-sized oligomers, however, appears to facilitate toxicity causing neuronal injury. Such aggregation may occur due to $\mathrm{A} \beta$ over-production as in early onset $\mathrm{AD}$ patients (age 65 or younger) with APP-related gene mutations, or in the late onset AD patients, diminished ability to remove $\mathrm{A} \beta$ as a result of ageing promotes the assembling of $\mathrm{A} \beta$ into larger oligomers and fibrillar plaques.

While there is evidence showing a robust correlation between the soluble oligomer levels and the extent of synaptic loss and cognitive impairment, the density of the fibrillar plaques detected does not always correlate with the severity of dementia [4,5]. The pathogenic relevance of $\mathrm{A} \beta$ is evident nevertheless and therapeutic treatments have been developed focusing on clearing excess $\mathrm{A} \beta$, preventing its formation or removing it. These interventional strategies have so far failed to cure or halt the progression of the disease except for modest or transient benefit. AD pathology is also characterised by an inflammatory response which is driven by the brain's innate immune system, primarily the microglial cells. As the resident immune cells of the central nervous system (CNS), microglia, in its resting states, surveys the environment guarding CNS against foreign invasion or structural anomaly such as $\mathrm{A} \beta$ aggregates. Upon stimulation (by $\mathrm{A} \beta$ aggregates, for example) microglia react to trigger a cascade of events leading to the secretion of inflammatory mediators which necessarily promote physiological inflammatory response and, possibly, the production of neurotoxin for the clearance of the damaged neurones. Accordingly, down-regulation events will take place to reduce the inflammatory response and restore homeostasis. Chronic inflammation ensues if the regulatory mechanism fails to restore the homeostatic environment of the CNS, as is observed in $\mathrm{AD}$, resulting in the over production of inflammatory mediators and the increased production of $\mathrm{A} \beta$ by the damaged neurone cells; the positive feedback of $\mathrm{A} \beta$ on the already stimulated immune state further exacerbates the progression of the disease. Because of its ability to stimulate microglia, the role of amyloid $\beta$, causative or secondary, remains relevant and significant in AD pathology. This motivates us to propose a mathematical model to examine the mechanisms involved in the clearance of the various $\mathrm{A} \beta$ related proteins and the subsequent effect on their accumulations, in particular, the buildup of $\mathrm{A} \beta$ plaques. 
Various in vitro experiments have shown that $\mathrm{A} \beta$ self-associates to form assemblies of different sizes (see, for examples, $[3,6,7]$ ) and mathematical models have been proposed to model its dynamic polymerisation. Stepwise polymerisation based on the discrete form of Smoluchowski equations, see for example $[8,9,10]$, leads to a very broad range of size/weight distribution that is amenable to numerical solutions but precludes mathematical analysis. Other models assume fewer $\mathrm{A} \beta$ related species [11, 12, 13]; Helal et al. [12], for example, considered only $\mathrm{A} \beta$ oligomers and plaques whereas Ciuperca et al. [13] further included $\mathrm{A} \beta$ fibrils and both models incorporated the role of prions to investigate the progression of the disease. In the current study, we propose a polymerisation scheme by dividing all $\mathrm{A} \beta$ related proteins and aggregates into five different categories as suggested in the review by Heppner et al. [6], whereby the binding of these proteins will either result in the length/size increase of existing proteins or the creation of new proteins in the lager size category. Our focus here is on the clearance and the locality of the buildup of $\mathrm{A} \beta$ aggregates, in particular, how these results vary according to different mechanism of protein transport, namely, via diffusion or the circulation of the interstitial fluid (ISF) of the brain. The existence of ISF bulk flow has been suggested by Abbott [21] and Weller [22]. While diffusion could explain some aspects of exchange between CSF and the brain close to the boundary, it is inefficient at distances over a few millimetre (further inside the brain). With ISF providing one-third of CSF source (and the other two thirds from choroid plexus), ISF circulation can be expected to facilitate the clearance of $\mathrm{A} \beta$ into the surrounding CSF. Furthermore, $\mathrm{A} \beta$ plaques deposits have been reported to appear first in isocortical areas and later in the allocortex or limbic structures [23] which, as our simulations in Section 4 show, is not achievable without ISF circulation.

Alzheimers disease is a highly complex, multi-facetted progressive disease that develops over many years. The aim of the current study is centred on the formation of various $\mathrm{A} \beta$ species, their clearance through enzyme degradation, diffusion and ISF outflow, and the spatial distribution of aggregates that results by combining these factors; we are assuming that the many other aspects of AD are not directly involved here. There have been numerous modelling work on the many other aspects of AD, such as disease progression $[14,15,16,17$, $18,19,20]$, brain atrophy [18] and drug trials [19] (with further references therein); some of these ideas could be incorporated into the current model for future investigations.

The proposed model involves construction of a nonlinear PDE system for the A $\beta$ species and ISF flow. We first formulate a two dimensional PDE model in Section 2 where a circular geometry is used to describe the transverse section of the brain. In Section 3, steady state results of the corresponding ODE system and an asymptotic analysis are presented. Numerical simulations are presented in Section 4, in which we simplify the geometry further to a one-dimensional model in order to compare the results of various parameter values. Two-dimensional results with non-uniform boundary conditions are also presented in this section. A brief discussion of the results and conclusion are given in Section 5.

\section{Model formulation}

A simplistic two dimensional circular geometry is adopted to represent the transverse section of the brain, see Figure 1, which is surrounded by the cerebral spinal fluid (CSF) of the subarachnoid on the outside and by the CSF of the ventricles on the inside. The two ventricles are assumed circular and placed symmetrically about the horizontal and vertical 


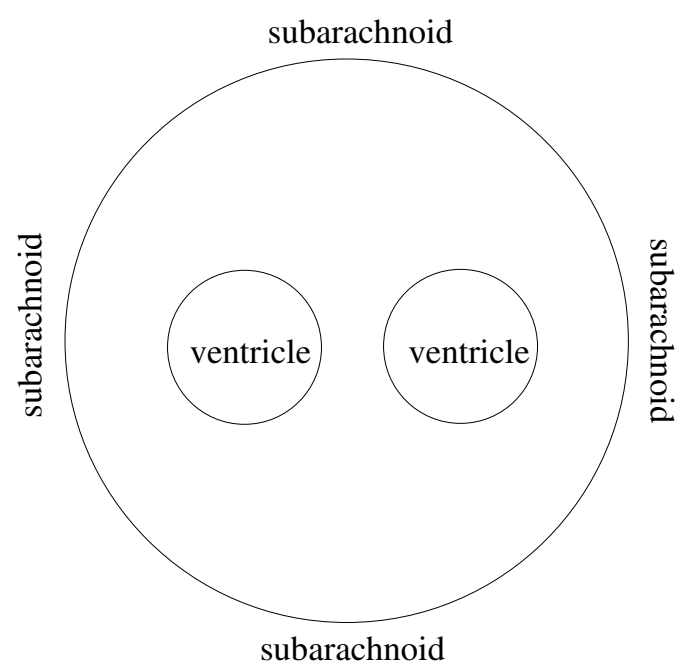

Figure 1: A two dimensional brain geometry, used in the simulation of Section 4.2 .

axes with the origin located at the centre of the brain. As a first approximation, a porous but homogeneous two-phase model is assumed for the brain region consisting of a solid phase that describes the brain tissue including the vasculature and a fluid phase for the interstitial fluid (ISF); we note that it is assumed the brain vasculature is uniformly spread over the domain. Amyloid $\beta$ monomers are assumed generated locally by neurone cells. The monomers and the subsequently formed oligomers are degraded enzymatically or drained into the surrounding CSF via ISF flow and diffusion. Although $\mathrm{A} \beta$ may be produced outside and transported into the brain, and, vice versa, reabsorbed into the blood vessels, the amount is likely to be negligible because of the existence of the blood-brain barriers (BBB) allowing very little exchange between blood and brain. Similar assumptions have been made by Bertsch et al.(2016) [8] regarding the clearance of $\mathrm{A} \beta$, furthermore, they have limited the transport of $\mathrm{A} \beta$ to that by diffusion only. The study by Abbott (2004) [21], however, found evidence for bulk flow of ISF with results showing that simultaneous removal of different molecular sizes is not achievable by diffusion alone. The assumption of ISF flow will therefore be incorporated in the current study. The modelling on the fluid phase, nevertheless, is simplified by assuming constant porosity with very little deformation taking place within the solid phase; consequently, we need only to consider the mass conservation and force balance of the fluid phase, namely,

$$
\phi \nabla \cdot v=S, \text { and } \quad v=-k \nabla p,
$$

where $v$ and $p$ are the fluid velocity and pressure respectively with $\phi$ denoting the constant volume fraction of ISF per unit volume, $k$ the permeability coefficient (assumed constant as $\phi$ is constant) and $S$ the rate constant of ISF source. Combing the two equations gives

$$
-\phi k \nabla^{2} p=S \text {. }
$$

Note that the source of ISF, which is assumed uniform throughout, is attributed mainly to the water from blood plasma across brain endothelium and that produced by brain cell metabolism. It has been suggested that CSF may recycle back through perivascular channels to rejoin the ISF flow, the contribution is generally assumed small and negligible as the recycled CSF would be composed of excretory product of the brain tissues, and thus will not be considered in the model. 


\begin{tabular}{|c|c|c|c|c|c|c|c|c|c|c|}
\hline $\mathrm{U}_{1}+\mathrm{U}_{1}$ & $\underset{\gamma 20}{\stackrel{\kappa 11}{\longrightarrow}}$ & $\mathrm{U}_{2}$ & $\mathrm{U}_{2}+\mathrm{U}_{2}$ & $\underset{\gamma 22}{\stackrel{\alpha 2 \kappa 22}{\rightleftarrows}}$ & $\mathrm{U}_{2}$ & $\mathrm{U}_{3}+\mathrm{U}_{3}$ & $\underset{\gamma 33}{\stackrel{\alpha 3 \kappa 33}{\underset{\gamma}{\rightleftarrows}}}$ & $\mathrm{U}_{3}$ & $\mathrm{U}_{4}+\mathrm{U}_{4}$ & $\underset{\gamma 44}{\stackrel{\alpha 4 \kappa 44}{\rightleftarrows}}$ \\
\hline $\mathrm{U}_{1}+\mathrm{U}_{2}$ & $\frac{\kappa 12}{\gamma 21}$ & $\mathrm{U}_{2}$ & $\mathrm{U}_{2}+\mathrm{U}_{2}$ & $\underset{\gamma 30}{\stackrel{\beta 2 \kappa 22}{\longrightarrow}}$ & $\mathrm{U}_{3}$ & $\mathrm{U}_{3}+\mathrm{U}_{3}$ & $\underset{\gamma 40}{\stackrel{\beta 3 \kappa 33}{\longrightarrow}}$ & $\mathrm{U}_{4}$ & $\mathrm{U}_{4}+\mathrm{U}_{4}$ & $\frac{\beta 4 \kappa 44}{\gamma 50}$ \\
\hline $\mathrm{U}_{1}+\mathrm{U}_{3}$ & $\frac{\kappa 13}{\gamma 31}$ & $\mathrm{U}_{3}$ & $\mathrm{U}_{2}+\mathrm{U}_{3}$ & $\frac{\kappa 23}{\underset{\gamma 32}{\longleftarrow}}$ & $\mathrm{U}_{3}$ & $\mathrm{U}_{3}+\mathrm{U}_{4}$ & $\underset{\gamma 43}{\stackrel{\kappa 34}{\longleftarrow}}$ & $\mathrm{U}_{4}$ & $\mathrm{U}_{4}+\mathrm{U}_{5}$ & $\frac{\kappa 45}{\gamma 54}$ \\
\hline $\mathrm{U}_{1}+\mathrm{U}_{4}$ & $\underset{\gamma 41}{\stackrel{\kappa 14}{\longrightarrow}}$ & $\mathrm{U}_{4}$ & $\mathrm{U}_{2}+\mathrm{U}_{4}$ & $\frac{\kappa 24}{\gamma 42}$ & $\mathrm{U}_{4}$ & $\mathrm{U}_{3}+\mathrm{U}_{5}$ & $\frac{\kappa 35}{\underset{\gamma 53}{\stackrel{-1}{\longrightarrow}}}$ & $\mathrm{U}_{5}$ & & \\
\hline $\mathrm{U}_{1}+\mathrm{U}_{5}$ & $\underset{\gamma 51}{\frac{\kappa 15}{\gamma-\longrightarrow}}$ & $\mathrm{U}_{5}$ & $\mathrm{U}_{2}+\mathrm{U}_{5}$ & $\underset{\gamma 52}{\stackrel{\kappa 25}{\leftarrow} \rightarrow-5}$ & $\mathrm{U}_{5}$ & & & & & \\
\hline
\end{tabular}

Figure 2: The association and dissociation between the five species, namely, monomers $\left(U_{1}\right)$, oligomers $\left(U_{2}\right)$, protofibrils $\left(U_{3}\right)$, fibrils $\left(U_{4}\right)$ and plaques $\left(U_{5}\right)$.

The self-association of $\mathrm{A} \beta$ gives rise to aggregates of different sizes which are not equally well detectable and have been categorised varyingly by different studies $[3,6,11,7]$. Generally, $\mathrm{A} \beta$ monomers are observed to form intermediates such as oligomers and protofibrils, the aggregations of these intermediates subsequently form filaments or fibrils and eventually the plaque deposit. We adopt the description used by Heppner et al. [6] and divide the $\mathrm{A} \beta$ related species into five categories: monomers $\left(U_{1}\right)$, oligomers $\left(U_{2}\right)$, protofibrils $\left(U_{3}\right)$, fibrils $\left(U_{4}\right)$ and plaques $\left(U_{5}\right)$, with each category including not a distinct size protein but a range of them and all species being able to diffuse and move with ISF circulation except for the plaques. Here we have used the capital letters $U_{i}^{\prime}$ 's to denote the five different species whereas below small letters $u_{i}$ 's are used to denote the concentration (number of protein per unit volume of the extracellular fluid) of the corresponding species. The association and fragmentation involved between these different species are depicted in the schematic of Figure 2, where all reactions are taken to be reversible although the associations that result in plaque formation or elongation are likely to be irreversible. It is also assumed that all species can bind with each other to form a new protein of a larger size category (as in the binding of two monomers $U_{1}$ forming a $U_{2}$ ) or to elongate an existing one (as in the binding of $U_{1}$ and $U_{2}$ resulting in a larger $U_{2}$ ). However, while the binding of two monomers can only result in a dimer of $U_{2}$ category, the association, for example, of two protofibrils $\left(U_{3}\right)$ of smaller size may result in a larger protofibril whereas that of two larger size $U_{3}$ are expected to form a new fibril $\left(U_{4}\right)$. The formation of a new protein, $U_{j+1}$ say, is only possible through the binding of two larger size $U_{j}$ 's which we assume to constitute $\beta_{j}$ proportion of such like-category binding and the other $\alpha_{j}$ proportion $\left(\alpha_{j}+\beta_{j}=1\right)$ results in larger size $U_{j}$ of the same category; it follows that the possibility that the association of $U_{i}$ and $U_{j}, i<j$ may produce a new protein in the $U_{j+1}$ category is ignored.

Note that while all five species are allowed to interact with each other, the schematic in Figure 2 is by no mean exhaustive and other pathways are possible but not included, for example, the spontaneous conversion of more than two protein into a new one in a category of larger size assembly; however, for the possibility of further analysis, the model is kept at a minimum.

Ignoring diffusion and advection for the static plaques $U_{5}$, constant volume fraction $\phi$ and mass conservation requires the concentration distribution $u_{i}(\boldsymbol{x}, t), i=1,2, \cdots 5$, to satisfy 
the following equations

$$
\begin{aligned}
\frac{\partial u_{i}}{\partial t}+\nabla \cdot\left(\boldsymbol{v} u_{i}\right) & =D_{i} \nabla^{2} u_{i}+F_{i}(\boldsymbol{u}), \quad i=1,2,3,4 \\
\frac{\partial u_{5}}{\partial t} & =F_{5}(\boldsymbol{u})
\end{aligned}
$$

where $\boldsymbol{v}=-k \nabla p$ is the ISF velocity and $\boldsymbol{u}=\left(u_{1}, u_{2}, u_{3}, u_{4}, u_{5}\right)$. The rate functions $F_{i}(\boldsymbol{u})^{\prime} \mathrm{s}$ are formulated below according to the reactions described in Figure 2 with the addition of a degradation term $\delta_{i} u_{i}$ for each species that describes the combined effect of $\mathrm{A} \beta$ clearance through enzyme degradation and possible reabsorption by the blood vessels, thus

$$
\begin{aligned}
& F_{1}(\boldsymbol{u})=f(\phi)+\sum_{j=2}^{N} \gamma_{j, 1} u_{j}+2 \gamma_{2,0} u_{2}-\kappa_{1,1} u_{1}^{2}-\sum_{j=1}^{N} \kappa_{1, j} u_{j} u_{1}-\delta_{1} u_{1}, \\
& F_{i}(\boldsymbol{u})=\bar{\kappa}_{i-1} u_{i-1}^{2}+\sum_{j=i}^{N} \gamma_{j,} u_{j}+2 \gamma_{i+1,0} u_{i+1}-\bar{\kappa}_{i} u_{i}^{2}-\sum_{j=i}^{N} \kappa_{i, j} u_{i} u_{j}-\gamma_{i, 0} u_{i}-\delta_{i} u_{i}, \quad i=2,3,4, \\
& F_{5}(\boldsymbol{u})=\bar{\kappa}_{4} u_{4}^{2}-\gamma_{5,0} u_{5}-\delta_{5} u_{5},
\end{aligned}
$$

where $\bar{\kappa}_{i}=\beta_{i} \kappa_{i i}$ and $\beta_{1}=1$. The function $f(\phi)$ is the production rate function of $\mathrm{A} \beta$ monomers by cells and with the assumption of constant porosity, this will be treated as a constant and denoted by $f_{\phi}$. Both diffusion and advection are incorporated into the movement of $U_{1}-U_{4}$ with diffusivity assumed constant for simplicity. Note that the permeability coefficient may be made dependent of $U_{5}$ to simulate the blockage of ISF flow by the presence of fibrillar plagues.

The role of CSF is mainly ignored for now except on the boundaries and for simplicity, constant pressure within CSF is assumed both in the ventricles and in the subarachnoid space. The fluid exchange between ISF and CSF can occur both across the bulk of the ependyma lining the cerebral ventricles, and across the pial/glial layer at the surface of the brain facing subarachnoid space, as the studies by Abbott [21] has shown using tracers, we thus impose the boundary conditions that

$$
\boldsymbol{J}_{\boldsymbol{i}} \cdot \boldsymbol{n}=Q_{i} u_{i}, \quad \text { and } p=p_{s},
$$

on the outside boundary, denoted by $\partial \Omega_{s}$, where $p_{s}$ is the constant CSF pressure in the subarachnoid, whereas on the inside (ventricular) boundary $\partial \Omega_{v}$,

$$
\boldsymbol{J}_{\boldsymbol{i}} \cdot \boldsymbol{n}=Q_{i} u_{i}, \text { and } p=p_{v}
$$

with $p_{v}$ being the CSF pressure of the ventricles. The outward flux for species $U_{i}$ is given by $J_{i}=v u_{i}-D_{i} \nabla u_{i}, i=1,2,3,4$, with $n$ denoting the outward unit normal and $Q_{i}$ the corresponding mass transfer coefficient. As the turnover of CSF is a rapid process in comparison to $\mathrm{A} \beta$ accumulation, we assume that the concentration of $U_{i}$ in the surrounding CSF is negligible and, for simplicity, the normal flux for each species is taken to be proportional to the concentration $u_{i}$ on the inside boundary. Finally, to close the system, for the time evolution of $U_{i}{ }^{\prime}$ s, we impose the initial condition that

$$
u_{i}=0, \text { at } t=0 .
$$


Note that we have assumed that the size of the $\mathrm{A} \beta$ are locally negligible, which is reasonable in modelling the early stages of the $\mathrm{A} \beta$ accumulation in the brain. Whilst, this will remain reasonable for $U_{i}, i \leq 4$, it is less so for the later stages of $U_{5}$ accumulation, where its volume can effect the porosity of the brain tissue effecting the ISF. This is an area that we intend to look at in future work.

\subsection{Non-dimensionalisation}

Before we proceed to analyse these equations, it is convenient to write them in dimensionless form. Equations (2.1)-(2.7) and the initial and boundary conditions (2.8)-(2.10) define the brain model which is made dimensionless using the following rescaling

$$
t=\frac{L}{v_{0}} \widehat{t}, x=L \widehat{x}, v=v_{0} \widehat{v}, p=p^{* *}+p^{*} \widehat{p}, u_{i}=u_{0} \widehat{u}_{i}
$$

and

$$
v_{0}=\frac{p^{*} k}{L}
$$

where $L$ is the brain radius, $u_{0}$ some characteristic $\mathrm{A} \beta$ monomer concentration and the quantities with hats denote the rescaled variables. Here $\widehat{t}=1$ represents the time scale for the fluid to flow across the radius of the brain. For the rescaling of pressure $p$, we set $p^{*}=p_{v}-p_{s}$ and $p^{* *}=p_{s}$ assuming $p_{v}>p_{s}$ so that $\widehat{p}=0$ on the boundary $\partial \Omega_{s}$ facing the subarachnoid space and $\widehat{p}=1$ on the ventricular boundary $\partial \Omega_{v}$. In $2 \mathrm{D}$ simulations below, two special cases are considered when $p_{s}=p_{v}$ and when the boundary pressure is non-uniform, in those cases, the pressure $p^{*}$ will assume the value of a non-zero positive constant. Consequently, the scaled parameters are

$$
P e=\frac{v_{0} L}{D_{1}}=\frac{p^{*} k}{D_{1}}, \quad \widehat{S}=\frac{L^{2} S}{k \phi p^{*}}, \quad \widehat{D}_{i}=\frac{D_{i}}{D_{1}}, \quad \widehat{Q}_{i}=\frac{Q_{i}}{v_{0}},
$$

where $i=2,3,4$ and

$$
\widehat{f_{\phi}}=\frac{f_{\phi} L}{v_{0} u_{0}}, \widehat{\gamma}_{*, *}=\frac{\gamma_{*, *} L}{v_{0}}, \widehat{\mathcal{\kappa}}_{*, *}=\frac{\kappa_{*, *} L u_{0}}{v_{0}}, \widehat{\delta}_{*}=\frac{\delta_{*} L}{v_{0}} .
$$

Dropping the hats for brevity gives the following dimensionless system

$$
\begin{aligned}
\nabla^{2} p & =-S \\
\boldsymbol{v} & =-\nabla p \\
\frac{\partial u_{i}}{\partial t}+\nabla \cdot\left(\boldsymbol{v} u_{i}\right) & =\frac{D_{i}}{P e} \nabla^{2} u_{i}+F_{i}(\boldsymbol{u}), \quad \text { for } i=1 . .4 \\
\frac{\partial u_{5}}{\partial t} & =F_{5}(\boldsymbol{u})
\end{aligned}
$$


and the rate functions are given by

$$
\begin{aligned}
& F_{1}(\boldsymbol{u})=f_{\phi}+\sum_{j=2}^{N} \gamma_{j, 1} u_{j}+2 \gamma_{2,0} u_{2}-\kappa_{1,1} u_{1}^{2}-\sum_{j=1}^{N} \kappa_{1, j} u_{j} u_{1}-\delta_{1} u_{1}, \\
& F_{i}(\boldsymbol{u})=\bar{\kappa}_{i-1} u_{i-1}^{2}+\sum_{j=i}^{N} \gamma_{j, i} u_{j}+2 \gamma_{i+1,0} u_{i+1}-\bar{\kappa}_{i} u_{i}^{2}-\sum_{j=i}^{N} \kappa_{i, j} u_{i} u_{j}-\gamma_{i, 0} u_{i}-\delta_{i} u_{i}, \quad i=2,3,4, \\
& F_{5}(\boldsymbol{u})=\bar{\kappa}_{4} u_{4}^{2}-\gamma_{5,0} u_{5}-\delta_{5} u_{5}
\end{aligned}
$$

where $\bar{\kappa}_{i}=\beta_{i} \kappa_{i, i}$ and $\beta_{1}=1$ and the initial and boundary conditions are

$$
\begin{array}{ll}
\text { At } t=0, & u_{i}=0, \quad i=1,2, \ldots, 5, \\
\text { On } \partial \Omega_{s}, & p=0, \quad\left(v u_{i}-\frac{D_{i}}{P e} \nabla u_{i}\right) \cdot n=Q_{i} u_{i}, \quad i=1,2,3,4, \\
\text { On } \partial \Omega_{v}, \quad p=1, \quad\left(v u_{i}-\frac{D_{i}}{P e} \nabla u_{i}\right) \cdot n=Q_{i} u_{i}, \quad i=1,2,3,4 .
\end{array}
$$

\section{Steady state analysis}

To understand better the important dynamics of the PDE system, we examine first the steady states of the spatially homogeneous case, namely,

$$
\begin{aligned}
& 0=f_{\phi}+\sum_{j=2}^{N} \gamma_{j, 1} u_{j}+2 \gamma_{2,0} u_{2}-\kappa_{1,1} u_{1}^{2}-\sum_{j=1}^{N} \kappa_{1, j} u_{j} u_{1}-\delta_{1} u_{1}, \\
& 0=\bar{\kappa}_{i-1} u_{i-1}^{2}+\sum_{j=i}^{N} \gamma_{j, i} u_{j}+2 \gamma_{i+1,0} u_{i+1}-\bar{\kappa}_{i} u_{i}^{2}-\sum_{j=i}^{N} \kappa_{i, j} u_{i} u_{j}-\gamma_{i 0} u_{i}-\delta_{i} u_{i}, \quad i=2,3,4, \\
& 0=\bar{\kappa}_{4} u_{4}^{2}-\gamma_{5,0} u_{5}-\delta_{5} u_{5},
\end{aligned}
$$

where $\bar{\kappa}_{i}=\beta_{i} \kappa_{i, i}$ and $\beta_{1}=1$. We are most interested in the plaque buildup as a result of slowing down in $\mathrm{A} \beta$ clearance (reflecting the problem of aging), and thus all parameters are fixed for the simulations and analysis below with the exception of the degradation rate constants $\delta_{i}{ }^{\prime}$ s. Furthermore, we expect larger size proteins are harder to clear and thus assume $\delta_{i+1}<\delta_{i}$; for convenience, we write $\delta=\delta_{5}$. As a generally rule, the backward reaction occurs at a slower rate compared to the corresponding forward reaction while the association rate of larger proteins (assuming more stable in its conformation compared to the smaller intermediates) is taken to be lower compared to the association of smaller proteins. As in Bertsch et al. [8], the rate constants assume the formulation

$$
\kappa_{i, j}=\frac{K}{(10 i-5) \times(10 j-5)^{\prime}}, \quad \text { and } \quad \gamma_{i, j}=\Gamma \kappa_{i j}
$$

where $K$ signifies the strength of the reaction and $\Gamma<1$. As the fragmentation of $U_{5}$ are less likely to occur, the rate constant $\gamma_{5, i}=0$ is assumed for all $i$.

Numerical simulations using Newton's method and a simple continuation scheme show the solutions of the above system, equations (3.24)-(3.26), as the degradation rate $\delta$ varies 


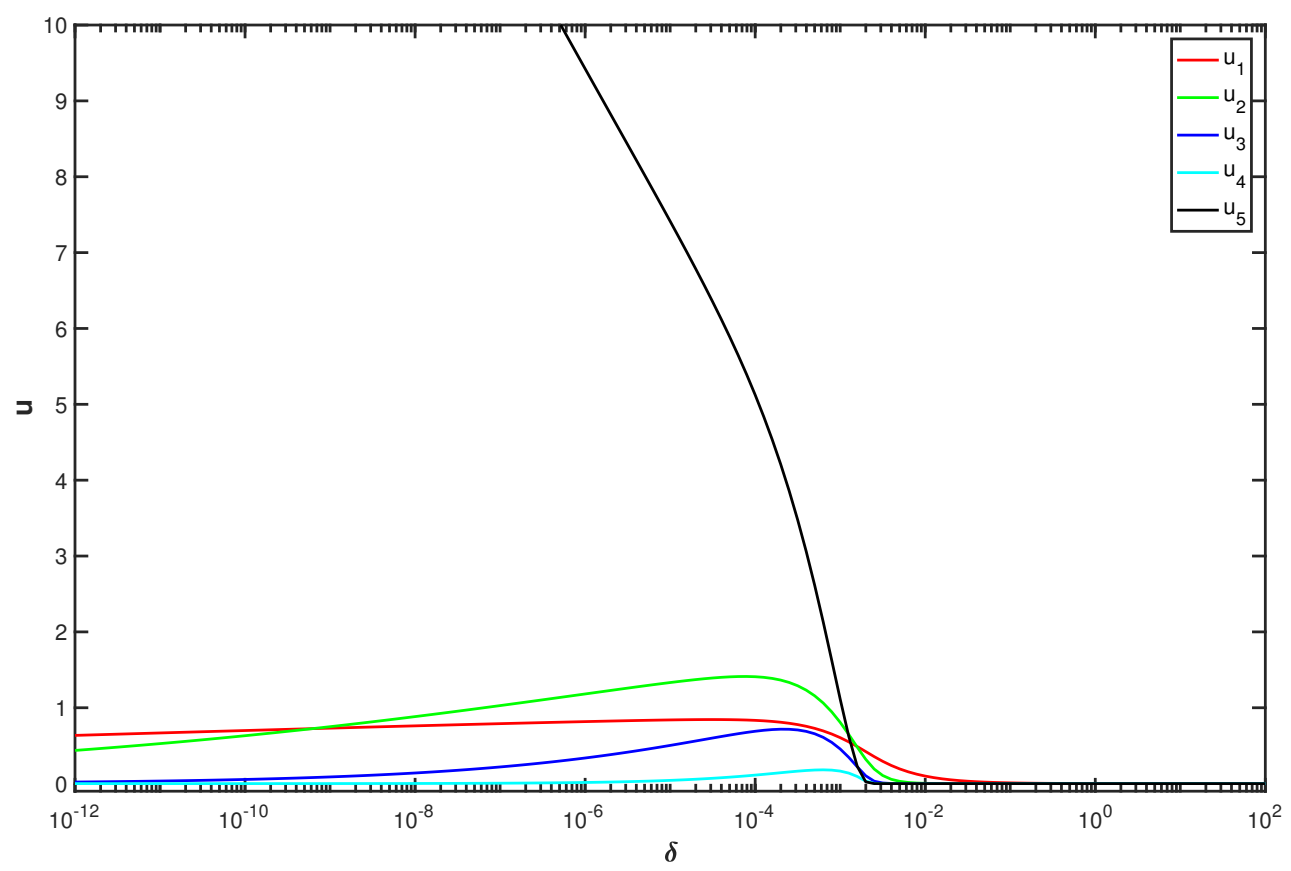

Figure 3: Simulations of equations (3.24)-(3.26) showing steady state solutions versus clearance/degradation rate $\delta$ with $\delta=\delta_{5}$ and $\delta_{i-1}=10 \delta_{i}$; other parameter values are listed in Table 1.

in Figure 3; the other parameter values used in the simulation are listed in Table 1. The concentration profiles of all species show that $u_{i} \rightarrow 0$ as $\delta \rightarrow \infty$, which can be expected that fast clearance prevents the buildup of all $\mathrm{A} \beta$ species; interestingly, however, as $\delta \rightarrow$ 0 all the intermediate species including monomers $U_{1}$ are maintained at relatively low concentration levels while the concentration of $U_{5}$ becomes increasingly large indicating that as the degradation rate $\delta$ becomes smaller, the large concentration of $U_{5}$ promotes more association with $U_{5}$ thus keeping other species at low concentrations.

To better understand the mechanism at play, we carry out a straightforward asymptotic analysis for small $\delta$ on a further reduced model by treating the three intermediates (oligomers, protofibrils and fibrils) as one species and divide all $\mathrm{A} \beta$ to three categories, namely, monomers $\left(U_{1}\right)$, oligomers $\left(U_{2}\right)$ and plaques $\left(U_{3}\right)$; the details are given in Appendix A. The steady state analysis of the reduced model will give insights to the five-species model, as the simulation (Figure 4) below shows that the reduced model still retains the characteristics of the fivespecies model.

\subsection{Asymptotical results for a reduced three species model}

The spatial homogeneous steady states of the reduced three-species model are given by, from Equations (A.1)-(A.3),

$$
\begin{aligned}
& 0=f_{\phi}-\left(\kappa_{1,1}+\bar{\kappa}_{1}\right) u_{1}^{2}-\kappa_{1,2} u_{1} u_{2}-\kappa_{1,3} u_{1} u_{3}+2 \gamma_{2,0} u_{2}+\gamma_{2,1} u_{2}-\delta_{1} u_{1} \\
& 0=\bar{\kappa}_{1} u_{1}^{2}-\left(\kappa_{2,2}+\bar{\kappa}_{2}\right) u_{2}^{2}-\kappa_{2,3} u_{2} u_{3}-\gamma_{2,0} u_{2}+\gamma_{2,2} u_{2}-\delta_{2} u_{2} \\
& 0=\bar{\kappa}_{2} u_{2}^{2}-\delta_{3} u_{3}
\end{aligned}
$$




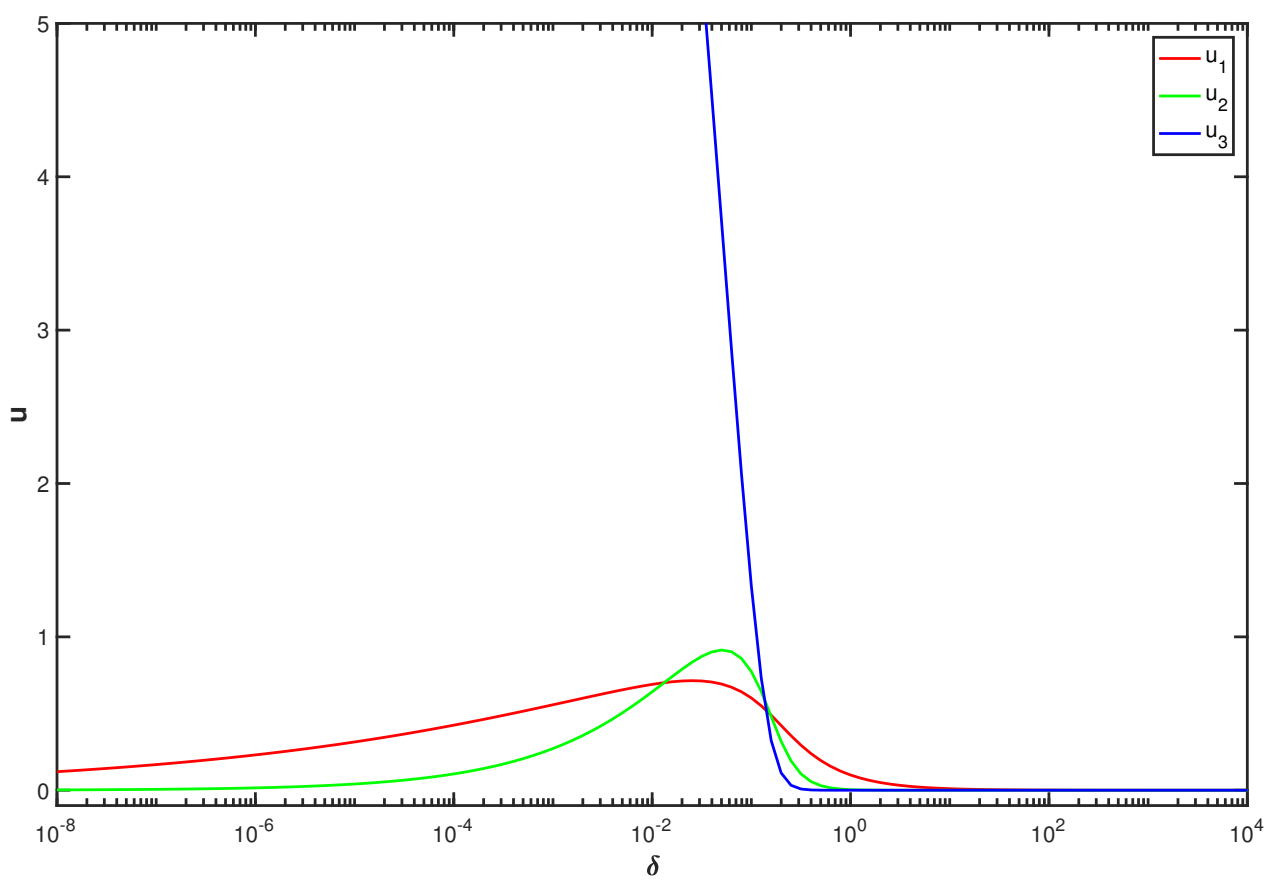

Figure 4: Simulations of equations (3.28)-(3.30) showing steady state solutions versus clearance/degradation strength $\delta$ with $\delta=\delta_{3}$ and $\delta_{i-1}=10 \delta_{i}$; other parameter values are given in Table 1.

where $u_{1}, u_{2}$ and $u_{3}$ are the concentrations of monomers, oligomers and plaques respectively. Here we have assumed $\gamma_{3, i}=0, \forall i$ with $\bar{\kappa}_{1}=\kappa_{1,1}$ and $\bar{\kappa}_{2}=\beta \kappa_{2,2}$. Numerical simulations of the above system, equations (3.28)-(3.30), showing the steady states versus the degradation rate $\delta$ are illustrated in Figure 4 with $\delta=\delta_{3}$ and $\delta_{i}<\delta_{i-1}$. Similar to the profiles in Figure 3 , all species $u_{i} \rightarrow 0$ as $\delta \rightarrow \infty$ which is to be expected for the effective clearance of A $\beta$ when $\delta$ is large; whereas, counterintuitively, as $\delta \rightarrow 0, u_{1}$ and $u_{2}$ stays at a relatively low levels while $u_{3}$ continues to build up. This suggests that relatively large concentration of the plaque and faster reaction rates promote the conversion of $U_{3}$ over degradation.

To confirm this result asymptotically for small degradation/clearance rate $\delta$, we rescale the variables with

$$
u_{1}=\delta^{a} \bar{u}_{1}, \quad u_{2}=\delta^{b} \bar{u}_{3}, \quad u_{3}=\delta^{2 b-1} \bar{u}_{3},
$$

where $a$ and $b$ are determined by balancing terms in equations (3.28)-(3.30). We subsequently find that $a=1 / 7, b=3 / 7$ with $2 b-1<0$ as expected for large $u_{3}$. Using the above rescaling in turn gives the leading order equations

$$
\begin{aligned}
& 0=f_{\phi}-\kappa_{13} \bar{u}_{1} \bar{u}_{3,}, \\
& 0=\bar{\kappa}_{1} \bar{u}_{1}^{2}-\kappa_{23} \bar{u}_{2} \bar{u}_{3}, \\
& 0=\bar{\kappa}_{2} \bar{u}_{2}^{2}-\delta_{3} \bar{u}_{3},
\end{aligned}
$$

and closed form solutions for $\bar{u}_{1}, \bar{u}_{2}$ and $\bar{u}_{3}$ are given by

$$
\bar{u}_{1}=\frac{f_{\phi}}{\kappa_{13} \bar{\kappa}_{2} \bar{u}_{2}^{2}}, \quad \bar{u}_{2}=\left(\frac{\bar{\kappa}_{1} f_{\phi}^{2}}{\bar{\kappa}_{2}^{3} \kappa_{13}^{2} \kappa_{23}}\right)^{1 / 7} \quad \text { and } \quad \bar{u}_{3}=\bar{\kappa}_{2} \bar{u}_{2}^{2} .
$$



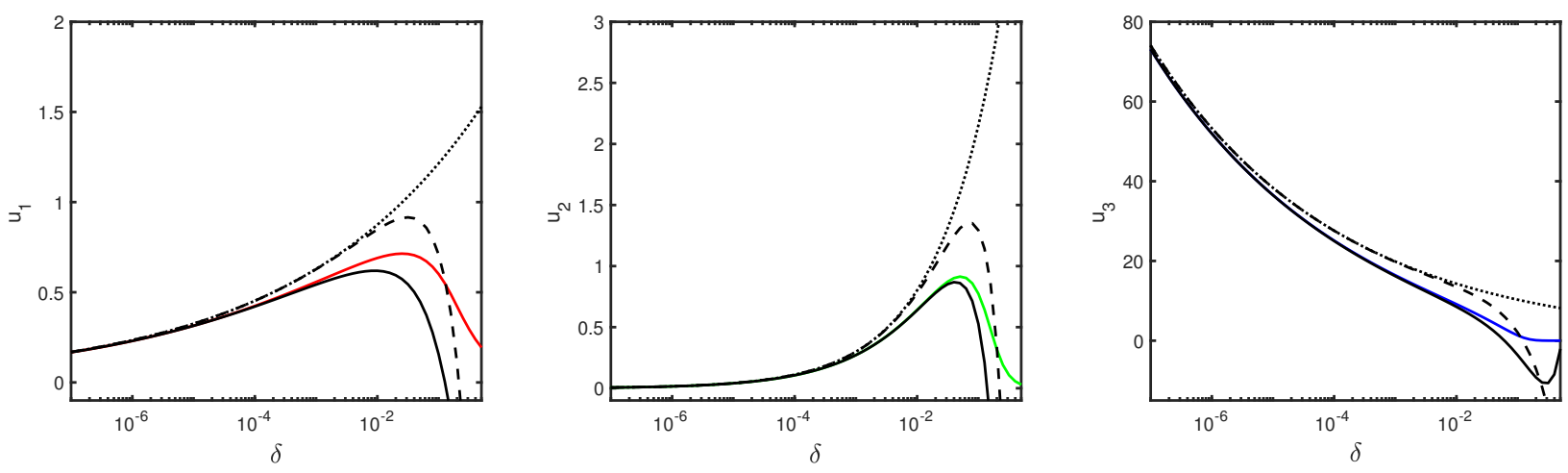

Figure 5: Comparison of the asymptotics with the exact solution of $u_{i}$ 's from Figure 4 with the exact solution $u_{1}$ in red, $u_{2}$ green and $u_{3}$ blue; leading order approximation $(\cdots)$, two terms approximation (- - , three terms approximation $(-)$. Parameter values are as given in Figure 4.

The leading order equations (3.32)-(3.34) suggest that while the clearance rate is small, the mechanisms at play are the production of $\mathrm{A} \beta$ and the formation of oligomers and plaques. The leading order term shows good agreement with the exact (numerical) result for very small $\delta$ but the asymptotic results continue to increase with $\delta$ while the exact numerical results decrease as $\delta$ increase, see Figure 5. We can improve on the asymptotics by evaluating the correction terms using an asymptotic series of the form

$$
\bar{u}_{i}=\sum_{j=0}^{\infty} \varepsilon^{j} U_{i j}, \quad \varepsilon=\delta^{1 / 7}, \quad \text { for } i=1,2,3 .
$$

Substituting (3.31) and (3.36) into the equations (3.28)-(3.30) gives the equations for the correction terms in the expansions, namely, $U_{11}, U_{21}$ and $U_{31}$, as

$$
\begin{array}{r}
\kappa_{13}\left(U_{10} U_{31}+U_{30} U_{11}\right)+\delta_{1} U_{10}=0, \\
\kappa_{23}\left(U_{20} U_{31}+U_{30} U_{21}-\left(r_{22}-r_{20}-\delta_{2}\right) U_{20}=0,\right. \\
2 \bar{\kappa}_{2} U_{20} U_{10}-U_{31}=0,
\end{array}
$$

where $U_{10}, U_{20}$ and $U_{30}$ are the leading order approximations given above by $\bar{u}_{1}, \bar{u}_{2}$ and $\bar{u}_{3}$ of Equation (3.35). The presence of the clearance terms in equations (3.37) - (3.39) result in the drop of $\mathrm{A} \beta$ monomer and oligomer levels for sufficiently large $\delta$ as observed in Figure 5 . The discrepancy between the asymptotic and the exact (numerical) solutions can be reduced further by taking additional correction terms in the expansion, namely, $U_{12}, U_{22}$ and $U_{32}$, satisfying equations

$$
\begin{aligned}
-2 \kappa_{11} U_{10}^{2}-\kappa_{13}\left(U_{11} U_{31}+U_{10} U_{32}+U_{30} U_{12}\right)-\delta_{1} U_{11} & =0 \\
\bar{\kappa}_{1}\left(U_{11}^{2}+2 U_{10} U_{12}\right)-\kappa_{23}\left(U_{21} U_{31}+U_{20} U_{32}+U_{30} U_{22}\right)+\left(r_{22}-r_{20}-\delta_{2}\right) U_{21} & =0 \\
\bar{\kappa}_{2}\left(U_{21}^{2}+2 U_{20} U_{22}\right)-U_{23} & =0
\end{aligned}
$$

The resulting three-term approximations are also illustrated in Figure 5 with solid curves.

We have performed similar asymptotic analysis on the above five species model and in this case, we found the leading order terms for the five species to be

$$
u_{1}=O\left(\delta^{1 / 31}\right), u_{2}=O\left(\delta^{3 / 31}\right), u_{3}=O\left(\delta^{7 / 31}\right), u_{4}=O\left(\delta^{15 / 31}\right) \text {, and } u_{5}=O\left(\delta^{-1 / 31}\right) \text {. }
$$


For the analysis to be effective, it is necessary that $\delta$ is very small and more terms in the expansions are needed for the asymptotic results to match well with the exact solutions. The leading order terms, however, suggest that, in the limit $\delta \rightarrow 0$, the concentration levels of the intermediate proteins are lower than those of the monomers and plaques, the concentration level (of the intermediate) decreases as the size of the protein increases. Note that linear stability analysis for each of these steady states results in a Jacobian with all negative eigenvalues indicating asymptotic stability of the solutions for all values of $\delta$.

Another characteristic of both reduced three- and five-species models is that a maximum exists for the intermediate size species, however, the values of $\delta$ where these maxima are located do not yield the maximum $u_{5}$. While we are not certain of the biological relevance of the range of $\delta$ investigated here, the results illustrated in Figure 3 and 4 lend support to the lack of correlation between plaque buildup and the severity of the disease, bearing in mind that it is the intermediate species that are toxic to the neuron cells and not the plaques nor the monomers. The continuous presence of these intermediate species are likely to induce microglia priming and contribute to the chronic inflammatory response. This results of small values of $\delta$ with large plaque concentration while intermediate species are maintained at low levels, show consistency with the report that patients with high plaque burden can be without dementia and with no evidence of neuroinflammation $[4,6,24]$.

\section{Numerical simulations of the PDE model}

To examine the effect of ISF outflow on $\mathrm{A} \beta$ clearance and the subsequent plague build up, we compare the result of diffusion-only case with that of diffusion and advection. As we are limited by the information available on the parameter values and their biological relevance, the simulations will be carried out for a range of values on some parameters. In this case, the results are better displayed as a one-dimensional (1D) problem for comparison purpose, which we will discuss immediately below. The two-dimensional (2D) simulations are carried out in the second part of this section where the results of non-uniform boundary conditions are presented. The parameter values used in the simulations are listed in Table 1 unless stated otherwise. The values of $D_{i}, Q_{i}$ and $\delta_{i}$ are assumed to decrease with protein size as we expect larger protein are harder to diffuse, transfer across the boundary and degrade respectively. We note that in the absence of many parameters values that can be sourced from the experimental literature, the dimensionless parameter values listed in Table 1 are largely assumed (with the formula of $\kappa_{i, j}$ taken from [8]), but reflective of reality (e.g. decreased diffusion coefficient with increased size in $\mathrm{A} \beta$ species) and tuned to produce results that are consistent with observations (e.g. [4, 21, 23]).

\subsection{One-dimensional simulations}

We first simplify the analysis by presenting 1D simulations of the model. This is done by treating the brain as a sphere and merging the two ventricles as another smaller sphere within the brain. Thus by assuming spherical symmetry, the model is reduced to a 1D problem, where $r=1$ is the outside boundary facing the subarachnoid and $r=r_{0}<1$ is the ventricular 
boundary on the inside. The governing equations (2.14)-(2.16) in 1D are

$$
\begin{aligned}
\frac{1}{r^{2}} \frac{\partial}{\partial r}\left(r^{2} \frac{\partial p}{\partial r}\right) & =-S, \\
v_{r} & =-\frac{\partial p}{\partial r}, \\
\frac{\partial u_{i}}{\partial t}+\frac{1}{r^{2}} \frac{\partial\left(r^{2} v_{r} u_{i}\right)}{\partial r} & =\frac{D_{i}}{P e} \frac{1}{r^{2}} \frac{\partial}{\partial r}\left(r^{2} \frac{\partial u_{i}}{\partial r}\right)+F_{i}(u), \quad \text { for } i=1 . .4,
\end{aligned}
$$

where $v=\left(v_{r}, 0,0\right)$ and the boundary conditions are

$$
\begin{array}{rll}
\text { at } \quad r=1, & p=0, \quad v_{r} u_{i}-\frac{D_{i}}{P e} \frac{\partial u_{i}}{\partial r}=Q_{i} u_{i}, & i=1,2,3,4, \\
\text { at } \quad r=r_{0}, & p=1, \quad v_{r} u_{i}-\frac{D_{i}}{P e} \frac{\partial u_{i}}{\partial r}=Q_{i} u_{i}, \quad i=1,2,3,4,
\end{array}
$$

with Equations (2.17)-(2.20) and the initial condition (2.21) unchanged.
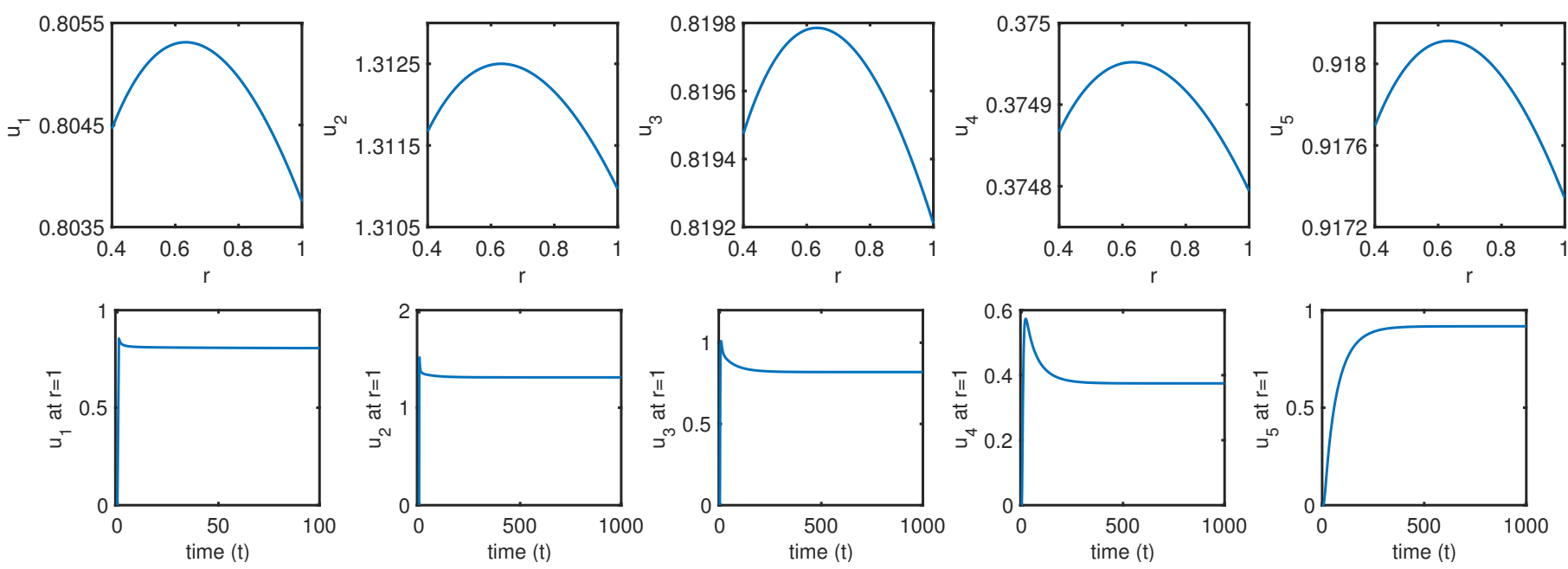

Figure 6: Simulations of the 1D model with diffusion only. The first row shows the distribution of $u_{i}$ 's at steady state; the second row shows the time plots of $u_{i}$ 's at the subarachnoid border $r=1$. The inside boundary $r_{0}=0.4$ is the ventricular boundary; other parameter values used in the simulations are listed in Table 1.

Figure 6 illustrates the result of diffusion only case ignoring the ISF flow. With the outward flux of $u_{i}$ 's attributed to diffusion only, i.e. $-\left(D_{i} / P e\right) \nabla u_{i}=Q_{i} u_{i}$, the concentration profile for each species changes very little throughout with slightly lower concentration nearer to the boundaries. Steady states have been attained as demonstrated by the time plots on the subarachnoid boundary $r=1$ (the bottom row). The smaller size oligomers $\left(U_{2}\right)$ appear to have the highest concentration among all the intermediate $\mathrm{A} \beta$ species. This is in broad agreement with experimental observations described in $[3,25]$ when investigating the assembly forms of the non-fribrillar $\mathrm{A} \beta$ assemblies isolated from human brain, the lower-n aggregates, ranging from dimers to octamers, are found to be the main contributor. Note that the accumulation of $\mathrm{A} \beta$ plagues are experimentally observed mainly in the isocortex (see [23] and the references therein), whereas the allocortex including hippocampus, the basal ganglia 


\begin{tabular}{|c|c|}
\hline Parameter & value \\
\hline$P e$ & 0.01 \\
$S$ & 20 \\
$D_{i}$ & $3^{-(i-1)}$ \\
$Q_{i}$ & $1 / 5^{i-1}, i=1,2,3,4$ \\
$f_{\phi}$ & 10 \\
$\kappa_{i, j}$ & $K /(10 i-5) *(10 j-5), \quad K=100$ \\
$\gamma_{i, j}$ & $\Gamma \kappa_{i, j}, \Gamma=1 / 10$ \\
$\gamma_{i, 0}$ & $\gamma_{i, i}$ \\
$\delta_{i}$ & $0.1 / 2^{i-1}$, \\
$\beta_{1}$ & 1 \\
$\beta_{2}, \beta_{3}, \beta_{4}$ & 0.5 \\
$r_{0}$ & 0.4 \\
\hline
\end{tabular}

Table 1: Table of dimensionless parameters.

and the cerebellum are involved in later stages and to a lesser extent than the isocortex. This observation does not agree well with the results of Figure 6 showing a maximum of the plaque concentration, $u_{5}$, away from the isocortex.

The concentration profiles of $\mathrm{A} \beta$ species are markedly different when the ISF flow is introduced as shown in Figure 7. Here $\mathrm{A} \beta$ plaque buildup mostly on the outside boundary thus agreeing with the isocortex accumulation in [23]; in fact all $\mathrm{A} \beta$ species accumulate in similar fashion, this is consistent with the pressure and velocity profiles illustrated on the bottom row of Figure 7, showing zero velocity nearer to the centre but increasing in magnitude toward the boundaries with positive velocity moving outwards and negative in the other direction. Both sets of results (of Figures 6 and 7) show improvement in
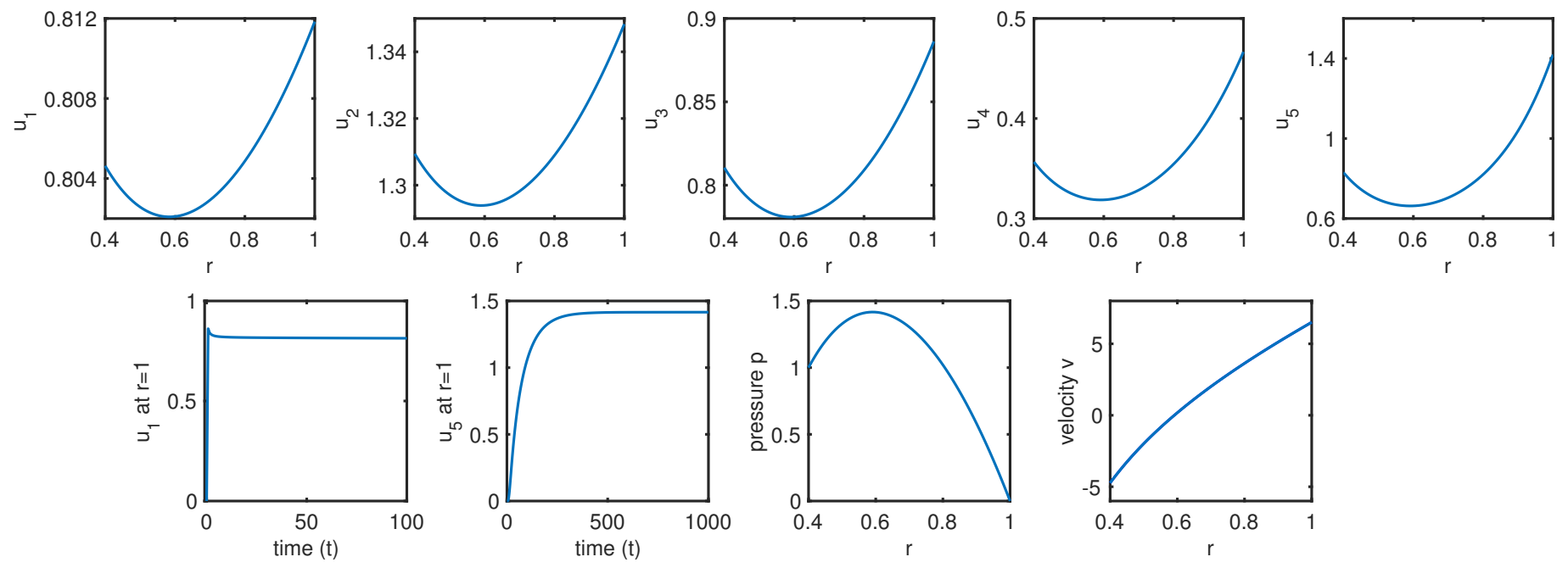

Figure 7: Simulations of the 1D model with diffusion and advection. The first row shows the distribution of $u_{i}$ 's at steady state; the second row shows the fluid velocity $v$ and pressure $p$, and the time evolution of $u_{1}$ and $u_{5}$ at the subarachnoid border. The inside boundary $r_{0}=0.4$ is the ventricular boundary; other parameter values used in the simulations are listed in Table 1. 

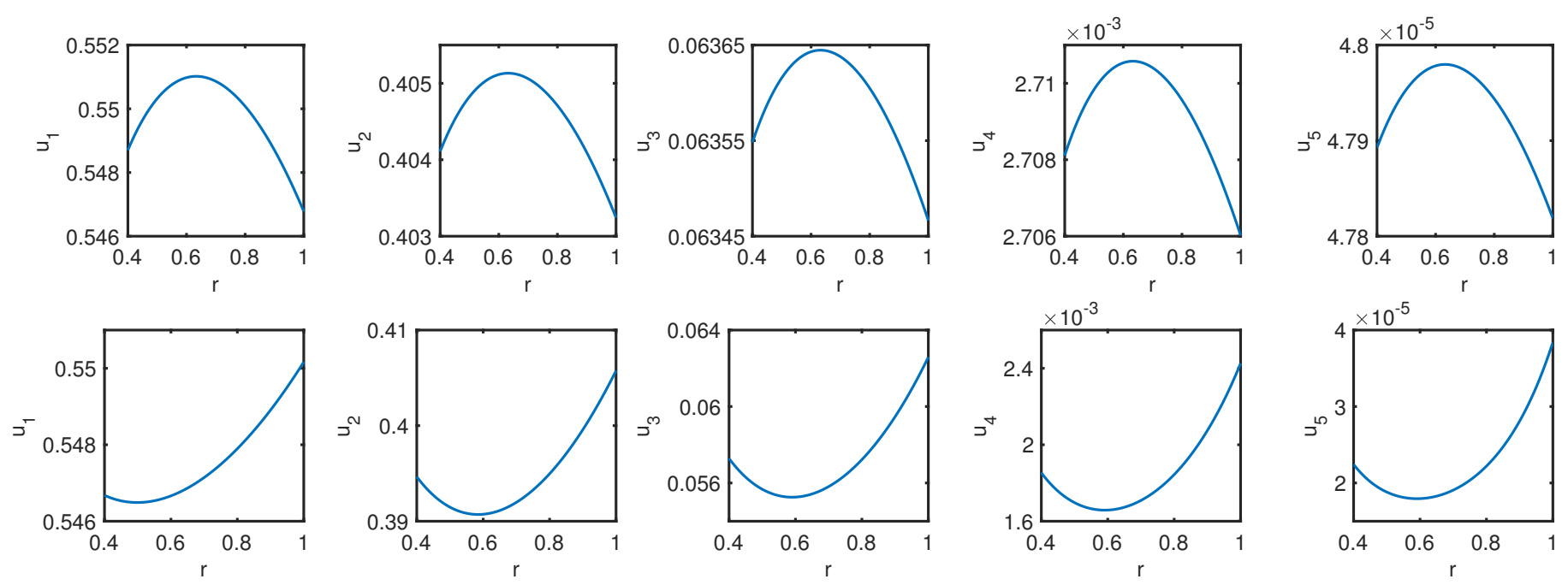

Figure 8: Simulations of the 1D model with mass transfer coefficient $Q_{i}=4 / 5^{i-1}, i=1,2,3,4$. The first row shows the steady state concentration profiles of $u_{i}{ }^{\prime}$ s for diffusion only case and on the bottom row those with both diffusion and advection. Other parameter values are listed in Table 1.

$\mathrm{A} \beta$ clearance when compared with the steady state solutions of the corresponding ODE system (Eqns (3.24)-(3.26), with parameters given in Table 1, give solutions $u_{1}=0.8959, u 2=$ $1.7155, u_{3}=1.2031, u_{4}=0.5555$ and $u_{5}=2.0152$ ) for which the diffusion and advection are both ignored. The additional presence of ISF (Figure 7), when compared with diffusiononly case (Figure 6), helped transport the protein to the boundaries, thus lowering the concentrations nearer to the centre of the brain while on the boundaries, the concentrations are higher. The effect of ISF circulation becomes more effective when the mass transfer coefficient $Q_{i}$ is increased (from $Q_{i}=1 / 5^{i-1}$ to $Q_{i}=4 / 5^{i-1}$ ) as the simulations of Figure 8 demonstrate; in this case, the clearance of $\mathrm{A} \beta$ is improved through both diffusion and ISF circulation with the concentrations further reduced when ISF flow is included. Note that, with $Q_{5}=0$, the lower concentration of $U_{5}$ is a result of reduced formation rate following the reduction in the concentrations of the smaller species; likewise, we expect the reduction in the concentrations of the smaller species to be attributed to both reduced formation rate and transport clearance. In general, the steady state concentration $u_{i}$ decreases with increasing $Q_{i}$ for all species and the effect of ISF flow becoming more effective as $Q_{i}$ increases, see Figure 9 for the concentrations of $U_{1}$ and $U_{5}$; the simulated concentration profiles of the other $\mathrm{A} \beta$ species from Figure 9-14 can be found in the supplementary material. If $Q_{i}$ decreases, the steady states of the diffusion-only case tend to those of the ODE system whereas those with ISF flow tend to spatially non-uniform limits, as shown in Figure 10.

We have also carried out simulations by varying $f_{\phi}$, the constant production rate of $\mathrm{A} \beta$ monomers and the rate constant of ISF secretion $S$, the results are shown in Figure 11 and Figure 12 respectively. The increased production of $\mathrm{A} \beta$ monomers, as expected, leads to higher concentration of all $\mathrm{A} \beta$ species throughout the brain whereas the effect of increased ISF secretion is not as uniform spatially. The subsequent velocity increase (in magnitude) helps transporting $\mathrm{A} \beta$ to the boundaries thus reducing the concentration around the centre of the brain but fails to prevent the accumulation near the boundary (Figure 12) unless they can be readily transferred outside the brain. By increasing the mass transfer rate with $Q_{i}$ in 

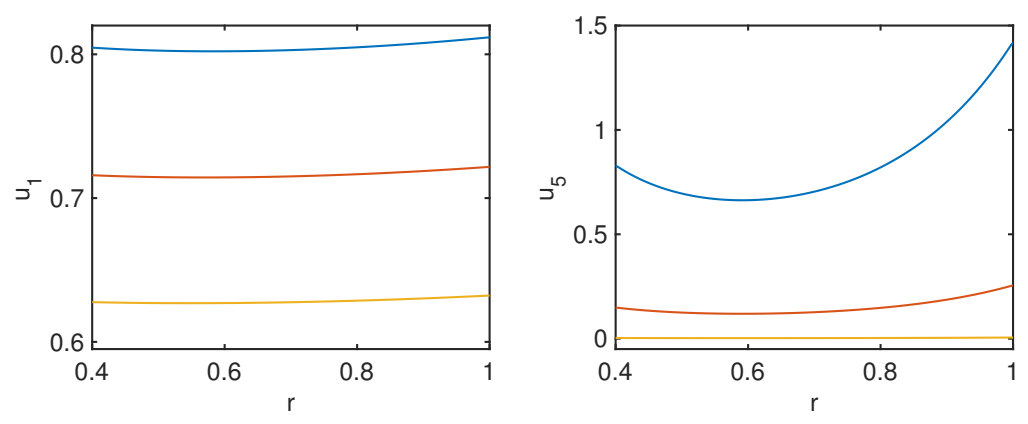

Figure 9: Simulations of the 1D model with mass transfer rate $Q_{i}=q / 5^{i-1}, q=1$ (blue), 2(red), 3(orange). The steady state concentration $u_{i}$ decreases with increasing $Q_{i}$ for all species; the concentration profiles of the other $\mathrm{A} \beta$ species can be found in the supplementary material. Other parameter values are listed in Table 1.
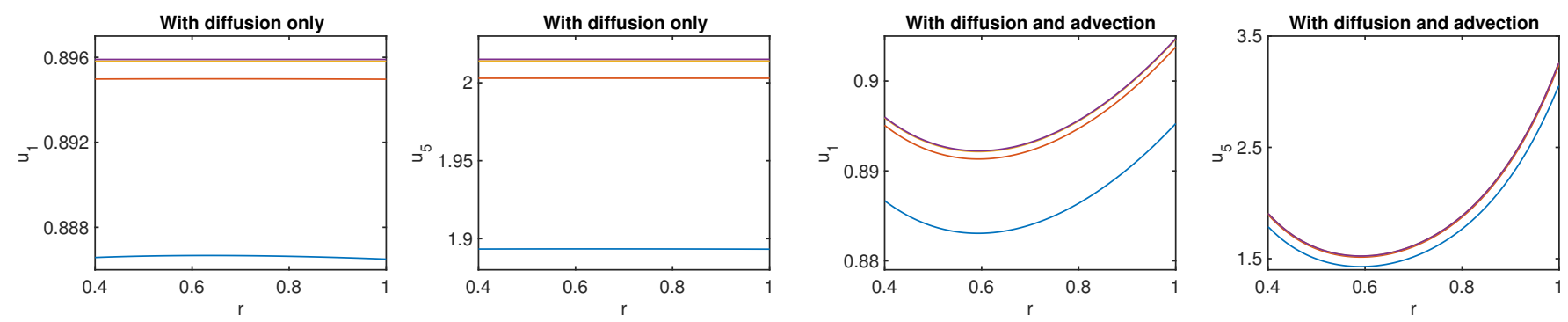

Figure 10: Simulations of the 1D model with mass transfer rate $Q_{i}=q / 5^{i-1}, q=0.1$ (blue), 0.01 (red), 0.001 (orange), 0.0001 (purple). The steady state concentrations of the diffusiononly cases approach those of the corresponding ODE system as $q$ becomes smaller whereas those with both diffusion and advection approach spatially non-uniform limits. The curves overlay each other as they approach their limits.
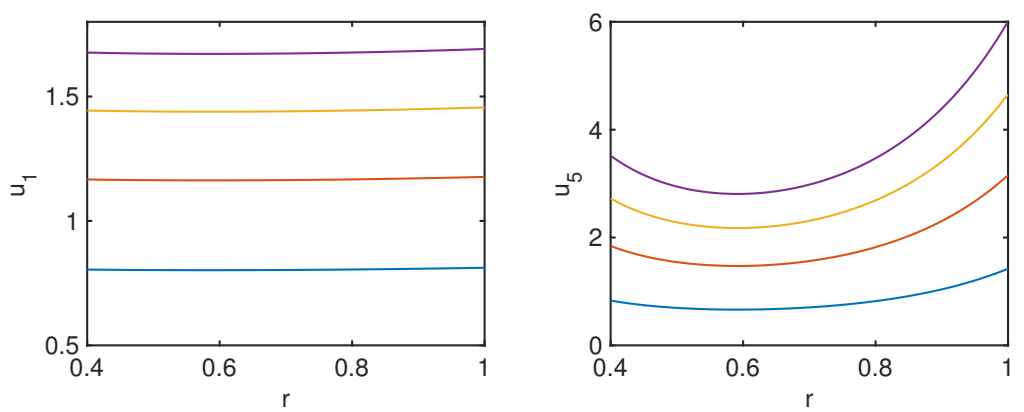

Figure 11: Simulations of the $1 \mathrm{D}$ model with $\mathrm{A} \beta$ monomers $U_{1}$ production rate constant $f_{\phi}=10$ (blue), 20 (red), 30 (orange), 40 (purple). The steady state concentration $u_{i}$ increases with increasing $f_{\phi}$ for all species as expected. Other parameter values are listed in Table 1. 
Figure 13, the increased ISF secretion can be seen to improve on the clearance throughout particularly for the larger $\mathrm{A} \beta$ species on the boundary.

Figures 12 and 13 indicate that the effectiveness of ISF circulation on A $\beta$ clearance also depend on the efficiency of protein transfer from inside the brain to the surrounding CSF. With our assumption that protein concentration in the surrounding CSF being negligible, the protein transfer rate varies at a fixed rate proportional to $\mathrm{A} \beta$ concentration on the inside boundary. However, decreasing CSF turnover is observed in the AD patients and also in elderly people in general, a consequence of lower production by choroid plexuses in the ventricles and reduced drainage through the subarachnoid sinus, leading to higher protein concentration level and subsequently slower protein transfer rate from ISF into CSF [1]. In cases when CSF protein concentration levels become comparable with $\mathrm{A} \beta$ concentration inside the boundary, we expect the normal flux to be proportional to the concentration difference across the boundary. As we have not specifically modelled CSF protein concentration in the current model, we approximate this effect by considering the modified boundary condition $J_{i} \cdot \boldsymbol{n}=Q_{i}\left(u_{i}-a u_{i}^{*}\right)$, with $u_{i}^{*}$ being the steady state concentrations of the corresponding ODE system, the results are shown in Figure 14. As $a$ increases, the protein concentration in the surrounding CSF increases, resulting in slower protein transfer rate across the brain boundary and subsequently higher $\mathrm{A} \beta$ concentration within the brain.

So far with the one-dimensional simulations, we have investigated and compared the effect of various factors (parameters) on $\mathrm{A} \beta$ concentration levels and distribution patterns. With the assumption of spherical symmetry, however, these factors are uniformly imposed in general when in reality non-uniformity is more apt to describe the brain environment. In the subsection below, similar analysis is carried out on two-dimensional simulations with the two dimensional brain geometry described in Figure 15, for which an immersed boundary formulation will be derived for Equations (2.14)-(2.17) using the indicator function, $H$, and transferring the boundary conditions to interface jump conditions. The $2 \mathrm{D}$ geometry allows us to impose non-uniform boundary condition which as we demonstrated below will result in hot spots of $\mathrm{A} \beta$ plaques accumulation on the boundary.
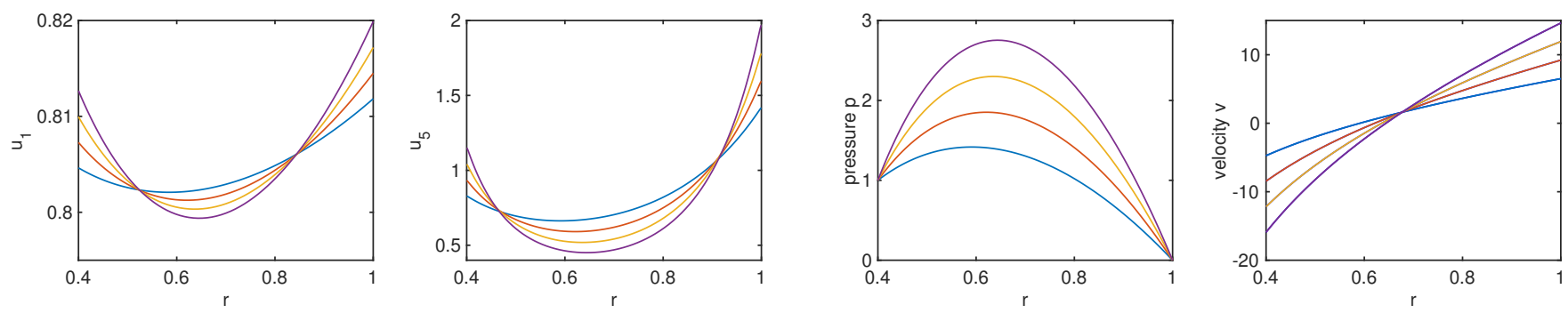

Figure 12: Simulations of the 1D model with ISF secretion rate constant $S=20$ (blue), 30 (red), 40 (orange) and 50 (purple) and $Q_{i}=q / 5^{i-1}, q=1$. The profiles of $u_{1}$ and $u_{5}$ show increased accumulation on the boundary as a result of increased ISF secretion rate. Such an increase in accumulation can be prevented if $\mathrm{A} \beta$ can be readily transported outwards as in Figure 13. 

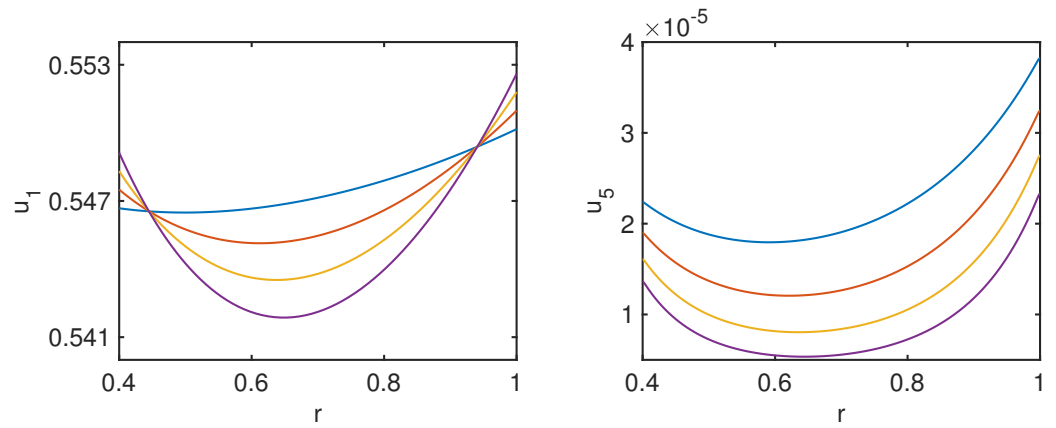

Figure 13: Simulations of the 1D model with $S=20$ (blue), 30 (red), 40 (orange) and 50 (purple) and $Q_{i}=q / 5^{i-1}, q=4$. The clearance of $\mathrm{A} \beta$ improves with increasing $q$.
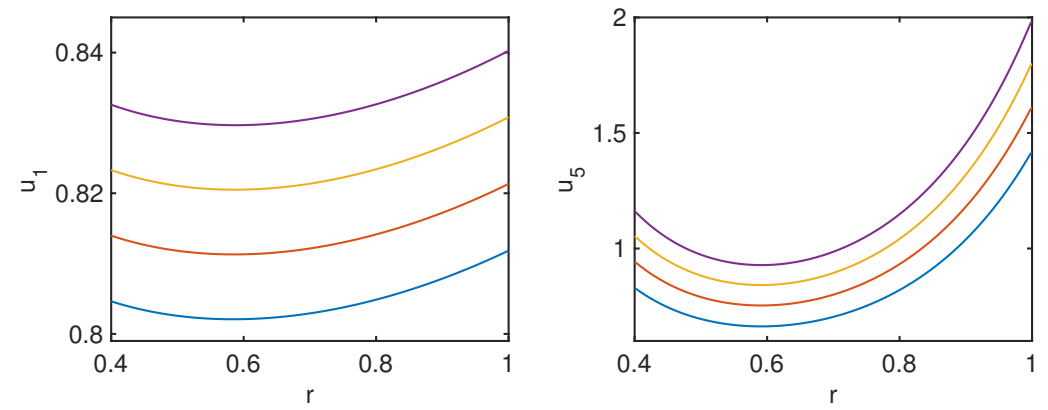

Figure 14: Simulations of the 1D model with the modified flux condition $J_{i} \cdot \boldsymbol{n}=Q_{i}\left(u_{i}-a u_{i}^{*}\right)$, with $u_{i}^{*}$ being the steady state solutions of the corresponding ODE system. The steady state concentrations increase with $a$ where $a=0$ (blue), 0.1 (red), 0.2 (orange) and 0.3 (purple). Other parameter values are listed in Table 1.

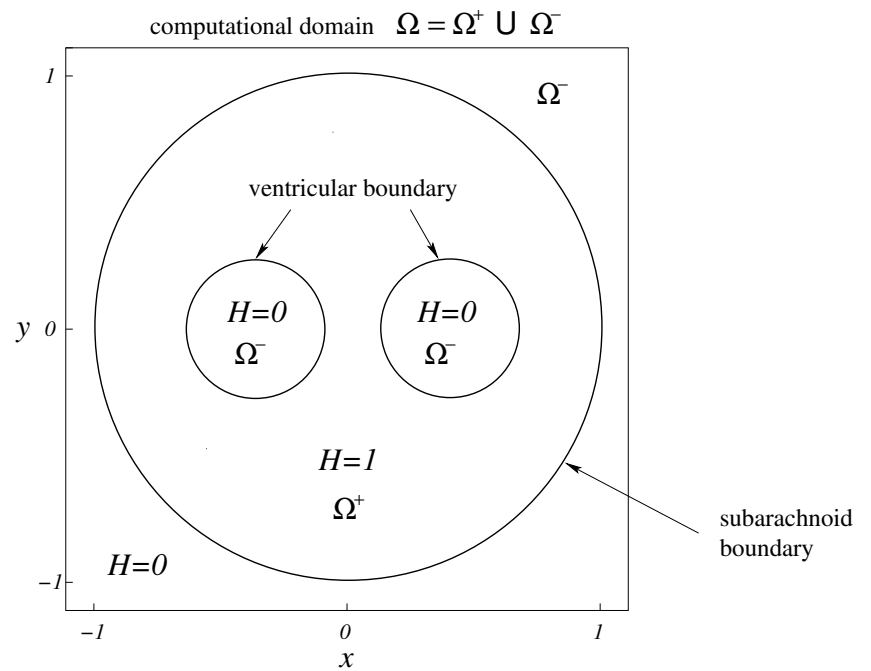

Figure 15: Schematic diagram of the computational domain. 


\subsection{The immersed boundary method for 2D simulations}

The immersed boundary method for Equations (2.14)-(2.17) are formulated and solved in a square computational domain as shown in Figure 15, where the brain outer boundary facing the subarachnoid and the inner ventricular boundary, denoted by $\partial \Omega^{+}$, are embedded in a square box. The division of the computational domain, $\Omega$, is facilitated using an indicator function $H$ so that $H=1$ on the inside of the brain $\left(\Omega^{+}\right)$and $H=0$ on the outside $\left(\Omega^{-}\right)$. We leave the detailed formulation of the method in Appendix B and focus on the simulated results below.

Figure 16 shows the distribution of $\mathrm{A} \beta$ concentrations as heatmaps for diffusion-only case whereas those including ISF flow are shown in Figure 17. The results here, as expected, are similar to $1 \mathrm{D}$ simulations that for the diffusion-only case, the spatial distribution of the concentrations show very little spatial variation and are lower near the boundaries; this is demonstrated on the bottom row of Figure 16, where the concentrations along the horizontal or vertical dashed-lines marked out with corresponding colors in the heatmaps are illustrated for each species, in particular, the red line runs along the axis $y=0.5$ for $-0.866 \leq x \leq 0.866$, the blue $y=0,-1 \leq x \leq 1$ and the green $x=0,-1 \leq y \leq 1$. Note that at time $t=1000$ the concentrations of all $\mathrm{A} \beta$ species have reached the steady states; the time plots of $u_{i}$ 's at a fixed location (see the supplementary material) are similar to those of 1D results of Figure 6. Parameter values used in the simulations are listed in Table 1 with the brain radius being one and ventricle radius 0.2 .
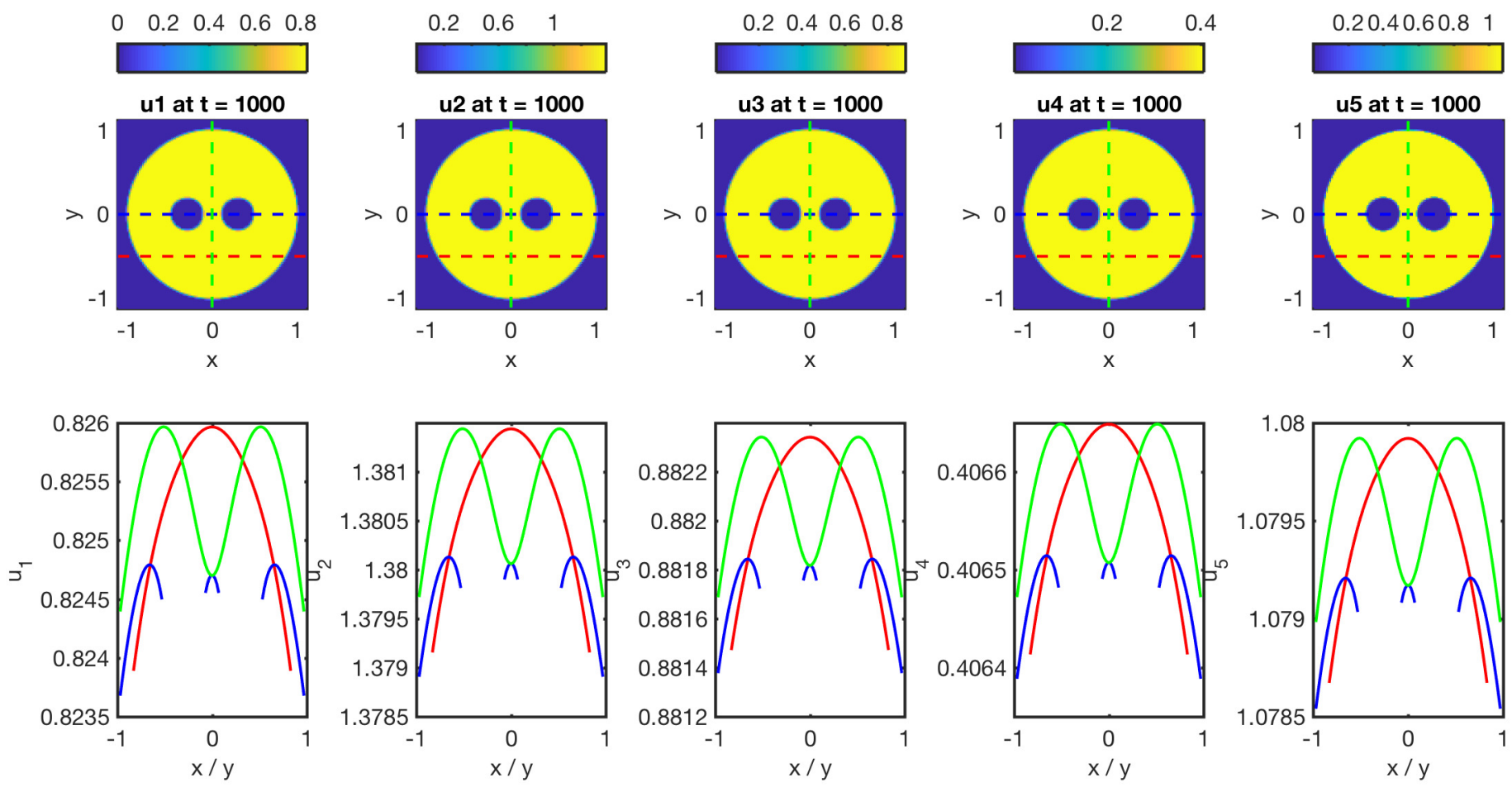

Figure 16: 2D simulations of the model with diffusion only. The heatmaps show the distribution of $u_{i}$ 's at steady states; on the second row, the concentrations of $\mathrm{A} \beta$ species are plotted along the horizontal or vertical dashed-lines marked out with the corresponding colors. Parameter values used in the simulations are listed in Table 1. 
The spatial variation of $\mathrm{A} \beta$ concentrations becomes more pronounced when the ISF flow is introduced, particularly for $u_{4}$ and $u_{5}$, see the heatmaps of Figure 17. Similar to the 1D results and contrary to those of the diffusion-only case, the concentrations are higher closer to the boundaries; this is consistent with the pressure and velocity fields shown in Figure 18. The concentration $u_{2}$ appears to be highest of all the soluble species both here and for the diffusion-only case, an observation also in agreement with 1D simulations. Note that we have assumed that fluid exchange is possible on both sides of the boundaries and the constant pressure in the ventricles $p_{v}$ is higher than that in the subarachnoid $p_{s}$ in accordance with the direction of CSF flow and, as a result, the concentrations of all $\mathrm{A} \beta$ species are higher closer to the outer boundary than the inner one, agreeing with the observations described by Serrano-Pozo et al. [23] that the accumulation of $\mathrm{A} \beta$ plaques will first appear closer to the outer boundary of the isocortex region. In cases of slowing down in CSF drainage as is often observed in elderly people and, particularly, in AD patients [1], the difference in the two pressures becomes small, for simplicity we assume $p_{s}=p_{v}, \mathrm{~A} \beta$ plaques can be seen in Figure 19 to accumulate closer to both side of the boundaries although the difference in the concentration is not as prominent as those in Figure 17.
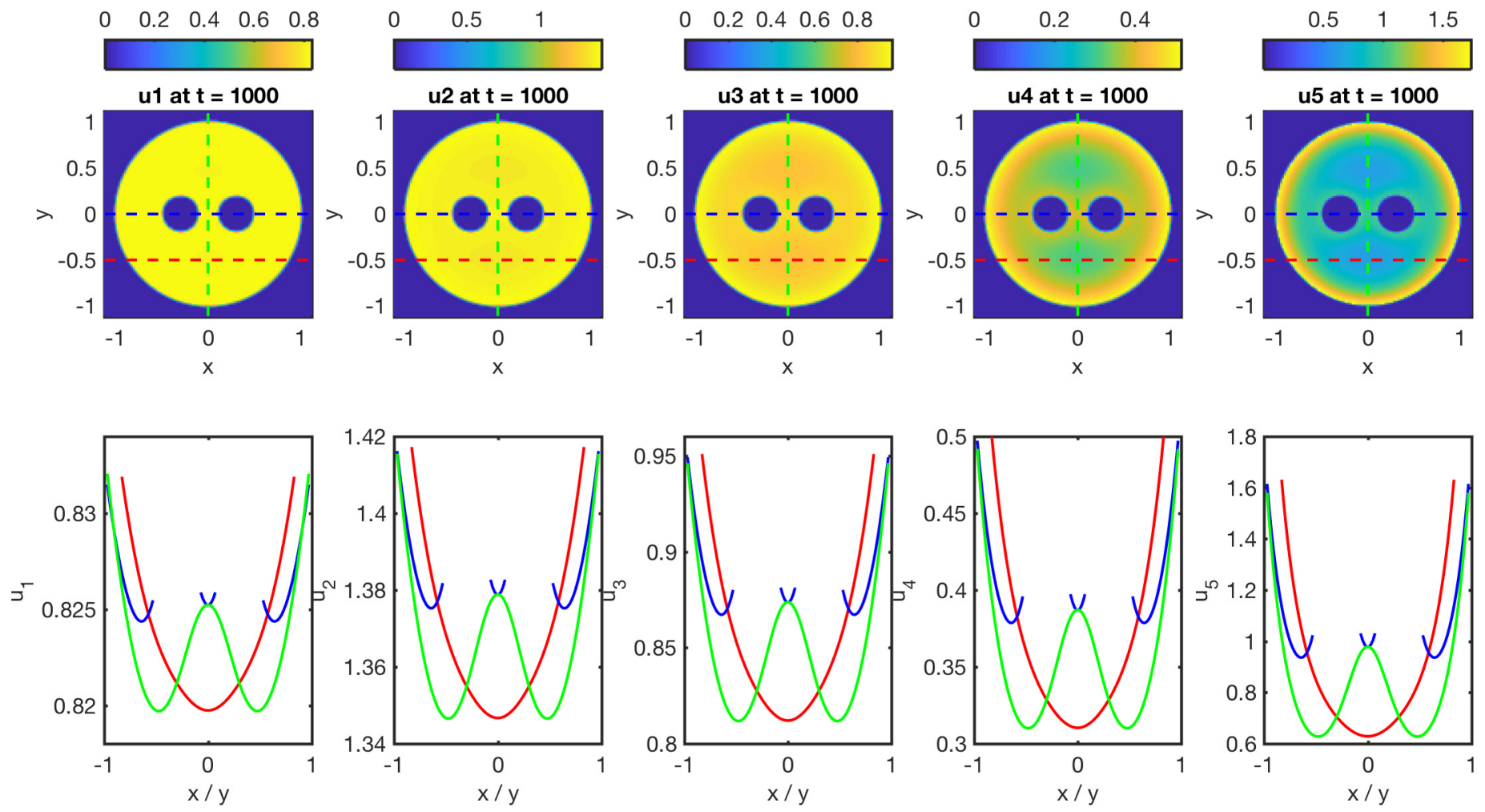

Figure 17: 2D simulations of the model with diffusion and advection. The heatmaps show the distribution of $u_{i}$ 's at steady states; on the second row, the concentrations are plotted along the horizontal (dashed) lines marked out with corresponding colors in the heatmaps. Parameter values are listed in Table 1.

For the purpose of our current study, we have treated the brain tissue as a uniform material with other properties (such as permeability) assumed constant for simplicity while 

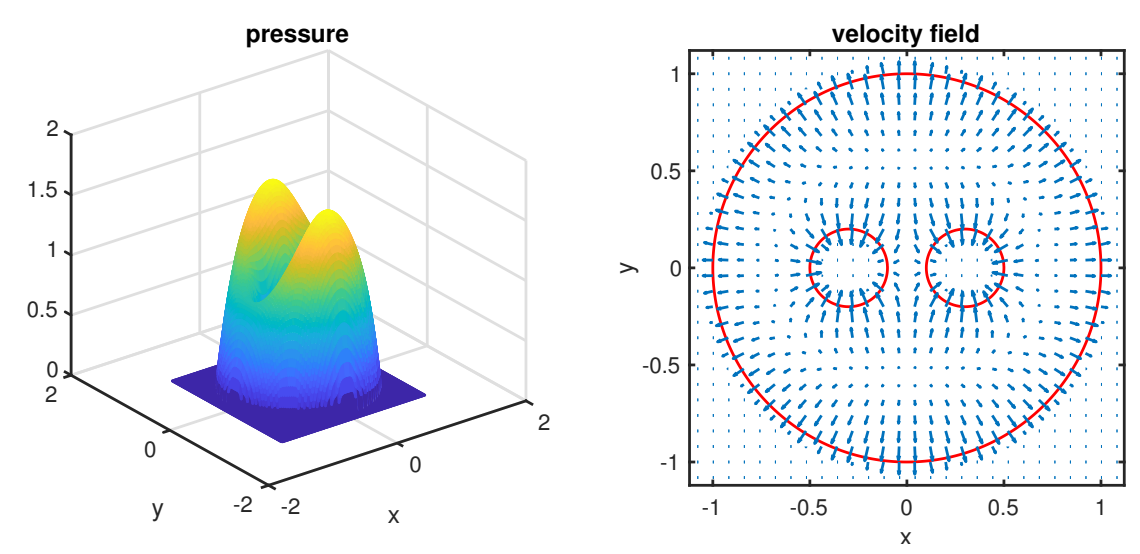

Figure 18: The corresponding pressure and velocity field of the 2D model with both diffusion and advection. Parameter values are listed in Table 1.
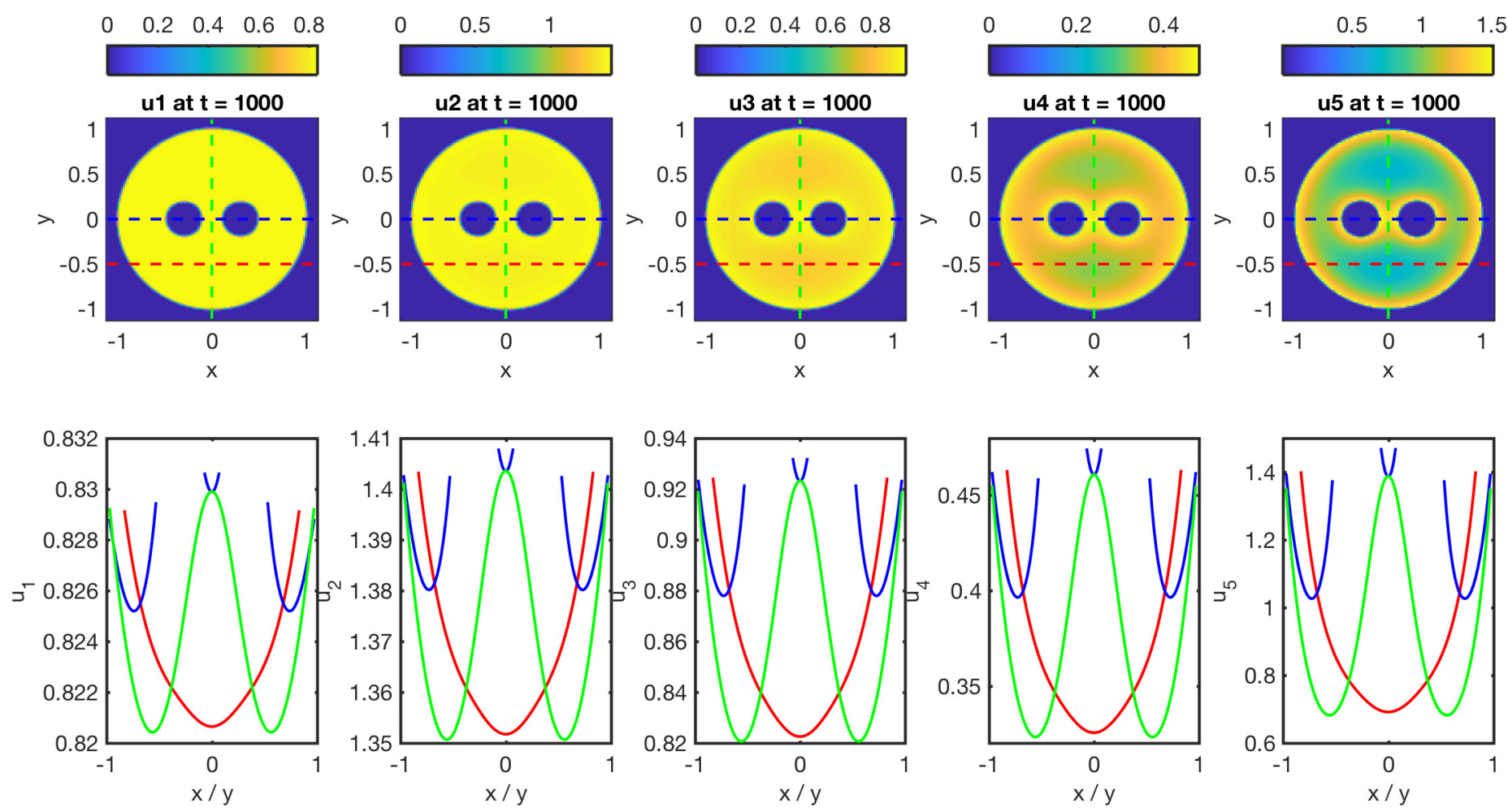

Figure 19: 2D simulations of the model with $p=0$ both on the inside and outside boundaries. The heatmaps show the distribution of $u_{i}$ 's at steady states with $\mathrm{A} \beta$ plaques $U_{5}$ accumulating near both sides of the boundaries; other parameter values are unchanged and as listed in Table 1.

imposing uniform boundary condition on both the inside and the outside boundaries. The human brain, however, is a complex and non-uniform construct, and so as an example to demonstrate the effect of this non-uniformity, we impose a periodic condition on the boundary facing the subarachnoid space by assuming $p=1 / 2+1 / 2 \sin (6 \theta)$ on the boundary $x=\cos \theta, y=\sin \theta, 0 \leq \theta \leq 2 \pi$ with $\theta=0$ along the positive $x$-axis; the simulations are 
shown in the first row of Figure 20. This non-uniform condition gives rise to clusters of A $\beta$ accumulation on the subarachnoid-facing boundary, particularly for $U_{4}$ and $U_{5}$ with the locations of these clusters situated at the sinks of $p$ on the boundarry. In the second row, we impose the periodic condition on both boundaries, the outside boundary is given as before and on the inside ventricular boundary, we set $p=1 / 2+1 / 2 \sin \left(3 \theta_{1}\right)$ on the right ventricle boundary $x-0.3=r_{1} \cos \theta_{1}, y=r_{1} \sin \theta_{1}$ with $r_{1}=0.2$ being the ventricle radius and $p=1 / 2+1 / 2 \sin \left(3 \theta_{2}+\pi / 2\right)$ on the left ventricle boundary $x+0.3=r_{1} \cos \theta_{2}, y=r_{1} \sin \theta_{2}$; the hot spots of $\mathrm{A} \beta$ accumulation are now also present on the inside boundary.
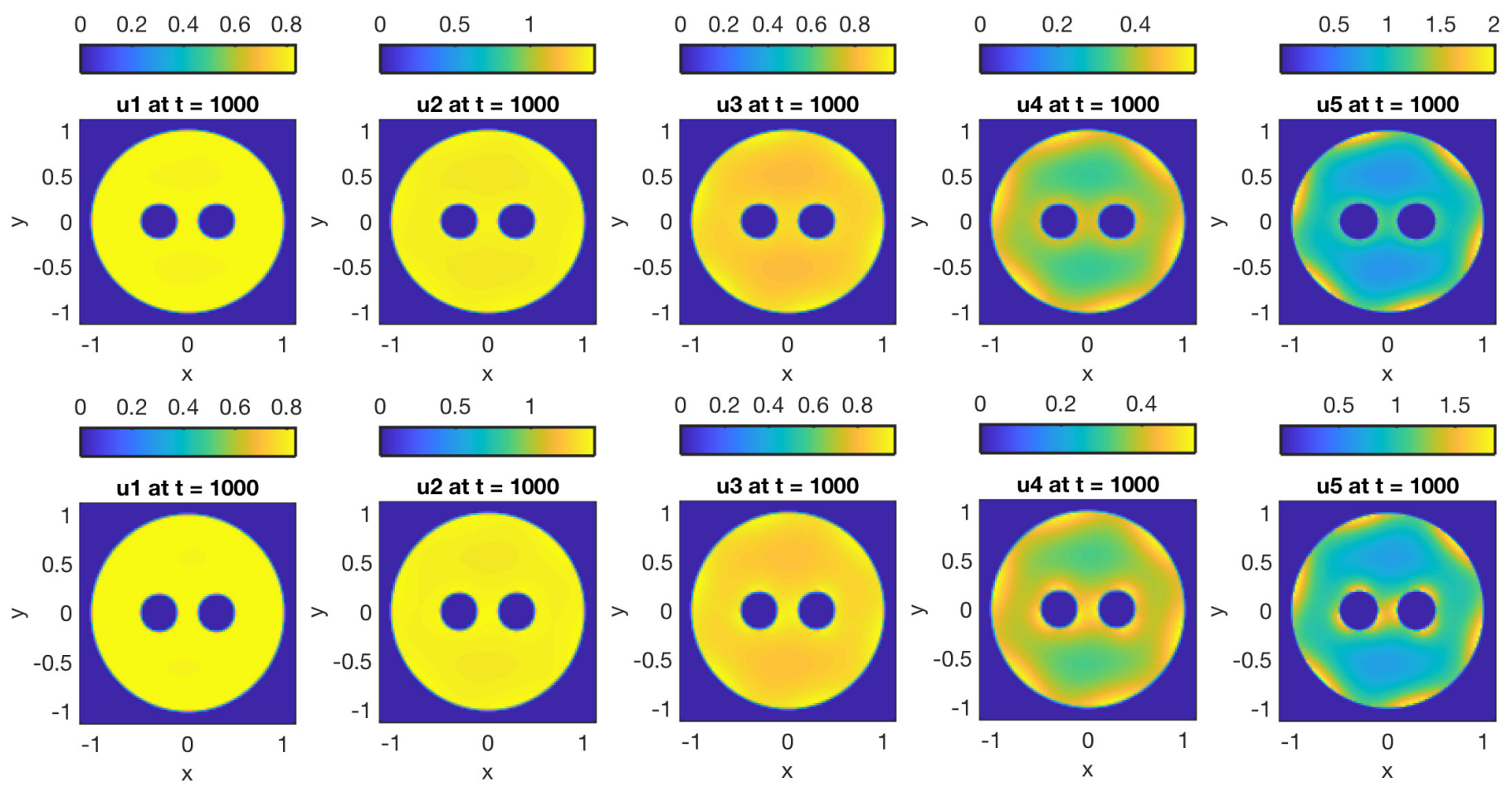

Figure 20: 2D simulations of the model with periodic boundary condition on the outside boundary for the top row and periodic conditions on both inside and outside boundaries on the bottom row. The heatmaps show clusters of $\mathrm{A} \beta$ accumulation, particularly of $U_{4}$ and $U_{5}$, on the boundaries; other parameter values are as listed in Table 1.

Note that we have, for simplicity, placed the two ventricles at central locations, symmetric about $x$ - and $y$-axis, in the above simulations. In cases when the ventricles are displaced from central locations, as the example of Figure 21 demonstrates, the hot spots of $\mathrm{A} \beta$ accumulation are similarly observed although the concentration distributions of $\mathrm{A} \beta$ species differ slightly from those with centralised ventricles in Figure 20. Likewise for the case of Figure 17 and Figure 19, if ventricles are shifted below the $x$-axis, $\mathrm{A} \beta$ still accumulates on the boundaries but the spatial distributions of $u_{i}$ 's are no longer symmetric about $x$-and $y$-axis. We have also carried out simulations with different values of $Q_{i}, S$ and $f_{\phi}$, the results are similar to those of 1D simulations and hence are not repeated here. 

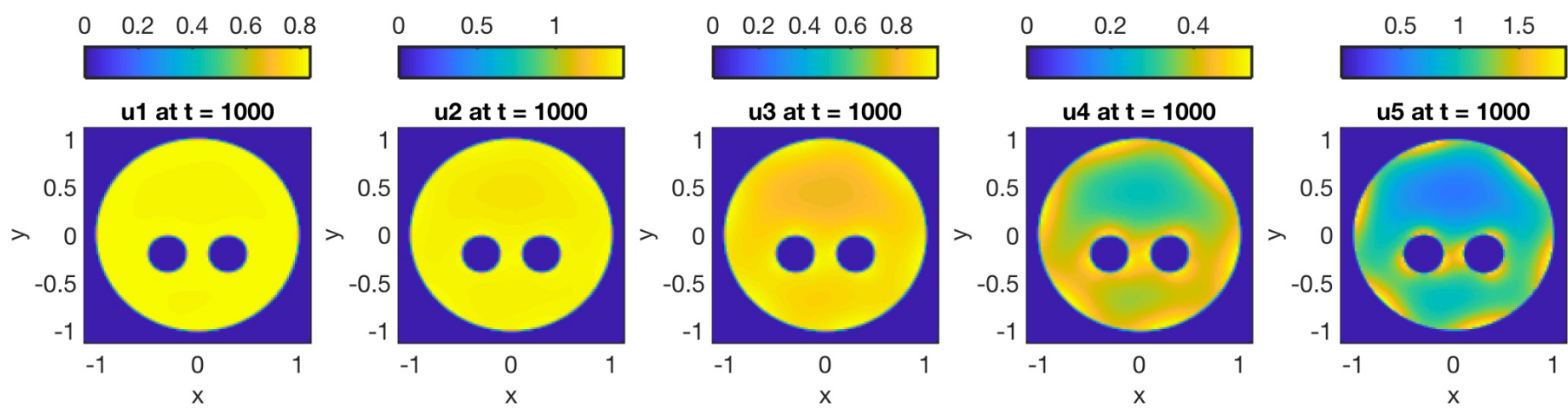

Figure 21: 2D simulations of the model with the centres of the two ventricles placed at $( \pm 0.3,-0.2)$. Periodic conditions are imposed on both inside and outside boundaries resulting in clusters of $\mathrm{A} \beta$ accumulation; other parameter values are as listed in Table 1.

\section{Conclusion and discussion}

In this paper, we have constructed a PDE model to study the formation and clearance of $\mathrm{A} \beta$ and the subsequent accumulation of $\mathrm{A} \beta$ plaque in the brain, which, for simplicity, is treated as a homogeneous porous medium. The model proposed here is different from the existing mathematical models (see [8] and the references therein) in that the polymerisation process that results in the formation of various $\mathrm{A} \beta$ species is made simpler by dividing all $\mathrm{A} \beta$ related proteins into five distinct categories as suggested by Heppner et al. [6], namely, monomers, oligomers, protofibrils, fibrils and plaques which are able to associate with each other forming larger proteins or breaking up into smaller sizes. A second new feature of the current model is the consideration of ISF circulation in the clearance and the subsequent accumulation of $\mathrm{A} \beta$. Under normal physiological conditions, these proteins are cleared mainly in three ways: through enzyme degradation, absorption into the capillaries or being transported into the surrounding CSF for drainage. These actions are modelled by assuming a degradation term in the equation for each species to account for the enzymatic action and capillary absorption whereas the drainage through CSF is facilitated through diffusion and ISF circulation for all species with the exception of the plaques.

We first carry out the steady state study of the spatial homogeneous system ignoring diffusion and advection, focusing on the buildup of $\mathrm{A} \beta$ as a consequence of slowing down in $\mathrm{A} \beta$ clearance, a phenomenon that is commonly associated with ageing. Steady state concentrations of the five $\mathrm{A} \beta$ species are shown to vary with the degradation rate constant and as expected, all species vanishes as the degradation rate constant tends to infinity, i.e. $u_{i} \rightarrow 0$ as $\delta \rightarrow \infty$; however, as $\delta \rightarrow 0$ all the intermediate species including the monomers are maintained at relatively low concentration levels while the plaque concentration continues to increase suggesting that as the degradation rate $\delta$ becomes smaller, the large concentration of $U_{5}$ promotes more association with $U_{5}$, helps maintaining other species at low concentrations. The increasing disparity between the concentration of the intermediate species and that of the plaque as $\delta \rightarrow 0$ also lend support to the lack of correspondence between the severity of the disease and the plaque buildup, bearing in mind that the intermediate $\mathrm{A} \beta$ species are toxic to neurone cells. Asymptotic expansions for small $\delta$ have been derived that provide analytic solutions to a reduced three-species model in which all $\mathrm{A} \beta$ are divided into three categories: monomers, oligomers and plaques. The leading order solution suggests that 
while the clearance rate is small, the dominant mechanisms are the production of $\mathrm{A} \beta$ and the formation of oligomers and plaques.

Numerical simulations are carried out for both one- and two-dimensional geometry, for which the one dimensional problem is a reduction from a three-dimensional brain structure by assuming radial symmetry. Two modes of protein transportation are considered: one with diffusion only and the other with diffusion and advection by means of ISF circulation. Distinct concentration profiles are obtained, in particular, when ISF flow is included, A $\beta$ plaque accumulations appear near the boundaries and much higher closer to the boundary facing the subarachnoid space, a result that is in agreement with the observation by SerranoPozo et al. [23]. For the one dimensional problem, we presented comparisons for different parameter values focusing on mass transfer coefficients $Q_{i}$, secretion rate constant for ISF, $S$ and the production rate of $\mathrm{A} \beta$ monomers, $f_{\phi}$. For $2 \mathrm{D}$ simulations, in particular, we have imposed periodic conditions on the boundaries where non-uniformities in the outflow of the ISF to CSF lead to clustering of $\mathrm{A} \beta$ on the boundaries.

With limited information available on the biological relevant parameter values, simulations are generated for different values of the same parameter while others held fixed to highlight the effects that are associated with the parameter. The simulated results indicate that while variations in the parameter values may have led to changes in the concentration levels but in generally do not alter $\mathrm{A} \beta$ distribution pattern, as shown in Figures 9-14, for which the presence of ISF is assumed and the accumulation of $\mathrm{A} \beta$ plaques closer to the boundary has been predicted. Note that the clearance of $\mathrm{A} \beta$ may be improved by increased ISF secretion provided the mass transfer coefficient is not too small (Figure 13); in the case when the mass transfer coefficient is small, the concentration is reduced near the centre whereas the accumulation closer to the boundaries becomes more pronounced with increasing ISF secretion (Figure 12).

Another factor that may lead to a reduction in $\mathrm{A} \beta$ transfer across the brain boundary is the increased protein level in the surrounding CSF. The example of Figure 13 demonstrates, by assuming different but constant $\mathrm{A} \beta$ concentrations in the CSF, that increased protein concentration in the CSF leads to a reduction in $\mathrm{A} \beta$ being transported across the brain boundaries, subsequently slowing down $\mathrm{A} \beta$ clearance within the brain. In addition to the increased protein level, the slowing down in CSF drainage also suggests the reduction in pressure difference between the ventricles and the subarachnoid, thus by setting $p=0$ on both boundaries in Figure 19, $\mathrm{A} \beta$ plaque accumulation can be observed on both sides of the boundaries.

To summarise, we have studied the association and disassociation of $\mathrm{A} \beta$, their clearance, and their distinct spatial distribution pattern according to different mechanisms of transportation. While $\mathrm{A} \beta$ aggregation remains a key component in the study of AD pathogenesis, the presence of them alone and particularly the plaque accumulation, can not explain the disease initiation nor its subsequent progression. Acting concomitantly with $\mathrm{A} \beta$ activities is the inflammatory response triggered by microglia, the resident immune cells of the central nervous system, as a response to any anomalies including neurone damages inflicted by toxic $\mathrm{A} \beta$ oligomers[6,29]. In cases when the subsequent down-regulation events fail to dampen the immune response, as most likely to have occurred in the AD patients, chronic inflammation may ensue, further exacerbating the disease. Our model in its current formulation is limited to the modelling of $\mathrm{A} \beta$ distribution and without explicitly considering neurones and other participants in the CNS, it is not yet fully equipped to study comprehensively the driving mechanisms of disease progression. It is therefore the topic of our on-going 
investigation to include neurones and immune cells into the current PDE model to provide a better understanding of the complex dynamics that could one day result in such a deleterious condition, becoming more prevalent as the population ages; such understanding, we hope, will facilitate in the development of effective therapeutic treatments to counter the progression of the disease.

Acknowledgements: The first author gratefully acknowledges the funding of MOST of Taiwan (MOST 106-2115-M-390-006). 


\section{References}

[1] Serot J-M, Zmudka J. and Jouanny P. A possible role for CSF turnover and choroid plexus in the pathogenesis of late onset Alzheimer's disease. J Alzheimers Dis (2012) 30, $17-26$.

[2] Swerdlow R.H. Pathogenesis of Alzheimer's disease. Clin Interv Aging (2007) 2(3), 347359.

[3] Walsh D.M. and Selkoe D.J. A $\beta$ Oligomers - a decade of discovery. J. Neurochem. (2007), 101, 1172-1184.

[4] Saraceno C., Musardo S, Marcello E., Pelucchi S and Di Luca M. Modeling Alzheimer's disease: from past to future. Front Pharmacol (2013) doi: 10.3389/fphar.2013.00077.

[5] Perl D.P. Neuropathology of Alzheimer's disease. Mt. Sainai J Med (2010) 77(1), 32-42.

[6] Heppner F.L., Ransohoff R.M. and Becher B. Immune attack: the role of inflammation in Alzheimer disease. Nat Rev Neurosci. (2015) 16(6):358-72. doi: 10.1038/nrn3880.

[7] Sadigh-Eteghad S, Sabermarouf B, Majdi A, Talebi M, Farhoudi M and Mahmoudi J. Amyloid-Beta: a crucial factor in Alzheimer's disease. Med princ Pract (2015) 24:1-10. doi:10.1159/000369101.

[8] Bertsch M., Franchi B., Marcello N., Tesi M.C., Tosin A. Alzheimer's disease: a mathematical model for onset and progression. Math Med Biol. (2016) doi: 10.1093/imammb/dqw003.

[9] Achdou Y., Franchi B., Marcello N. and Tesi M.C. A qualitative model for aggregation and diffusion of $\beta$-amyloid in Alzheimer's disease. J. Math. Biol.(2014) 67, 1369-1392.

[10] Franchi B. and Tesi M. C. (2013). A qualitative model for aggregation-fragmentation and diffusion of $\beta$-amyloid in Alzheimer's disease. Rend. Semin. Mat. Univ. Politec. Torino(2012) 70:75-84.

[11] Pallitto M.M. and Murphy R.M. A mathematical model of the kinetics of $\beta$-amyploid fibril growth from the denatured state. Biophy. J. (2001)81, 1805-1822.

[12] Helal M., Hingant E., Pujo-Menjouet L. and Webb G.F. Alzheimer's disease: analysis of a mathemtiacal model incorporating the role of prions. J. Math. Biol.(2014) 69, 1207-1235.

[13] Ciuperca I.S., Dumont M., Lakmeche A., Mazzocco P., Pujo-Menjouet L, Rezaei H. and Tine L.M. Alzheimer's disease and prion: an in vitro mathematical model. Discr. Contin. Dyn. Sys. B, to appear.

[14] Kuznetsov I.A. and Kuznetsov A.V. How the formation of amyloid plaques and neurofibrillary tangles may be related: a mathematical modelling study. Proc. R/ Soc. A (2018) 474:20170777.

[15] Kuznetsov I.A. and Kuznetsov A.V. Simulating the effect of formation of amyloid plaques on aggregation of tau protein. Proc. R/ Soc. A (2018) 474:20180511. 
[16] Weickenmeier J., Jucker M., Goriely A. and Kuhl E. A physics-based model explains the prion-like features of neurodegeneration in Alzheimer's disease, Parkin- son's disease, and amyotrophic lateral sclerosis. J. Mech. Phys. Solids (2019) 124: 264-281.

[17] Fornari S., Schäfer A., Jucker M., Goriely A. and Kuhl, E. Prionlike spreading of Alzheimer's disease within the brain's connectome. (2019) www.biorxiv.org/content/10.1101/529438.

[18] Schäfer A., Weickenmeier J., and Kuhl E. The interplay of biochemical and biomechanical degeneration in Alzheimer's disease. Comput. Methods Appl. Mech. Engrg. (2019) 352: 369-388.

[19] Hao W. and Friedman A. Mathematical model on Alzheimer's disease. BMC Syst. Biol. (2016) 10:108. doi:10.1186/s12918-016-0348-2.

[20] Puri I.K. and Li L. Mathematical modeling for the pathogenesis of Alzheimer's disease. PLoS ONE(2010) 5(12):e15176. doi:10.1371/journal.pone.0015176.

[21] Abbott N.J. Evidence for bulk flow of brain interstitial fluid: significance for physiology and pathology. Neurochem Int (2004) 45, 545-552.

[22] Weller R.O. Pathology of cerebrospinal fluid and Interstitial fluid of the CNS: significance for Alzheimer disease, prion disorders and multiple sclerosis. J. Neuropathology and Experimental Neurology (1994) 57(10), 885-894.

[23] Serrano-Pozo A, Frosch M.P., Masliah E. and Hyman B.T. Neuropathological alterations in Alzheimer Disease. Cold Spring Harb Perspect Med 2011;1:a006189

[24] Krstic D. and Knuesel I. Deciphering the mechanism underlying late-onset Alzheimer disease. Nat Rev Neurol (2013) 9(1):25-34.doi:10.1038/nrneurol.2012.236.

[25] Shankar G.M. and Walsh D.M. Alzheimer's disease: synaptic dysfunction and A $\beta$. Molecular Neurodegeneration (2009) 4:48. 2009, 4:48 doi:10.1186/1750-1326-4-48.

[26] C. S. Peskin, Flow patterns around heart valves: a numerical method, J. Comput. Phys.(1972) 10, 252-271.

[27] C. S. Peskin, The immersed boundary method, Acta Numerica, (2002) 1-39.

[28] C. S. Peskin and B. F. Printz, Improved volume conservation in the computation of flows with immersed elastic boundaries, J. Comput. Phys. (1993) 105, 33-36 .

[29] Chen W-W., Zhang X and Huang W-J. Role of neuroinflammation in neurodegenerative diseases. Mol Med Rep. (2016)133391-3396. 


\section{Appendix A: A three species model}

The three species model assumes all $\mathrm{A} \beta$ related proteins are devided into three categories: monomers $\left(U_{1}\right)$, oligomers $\left(U_{2}\right)$ and fibrillar plaques $\left(U_{3}\right)$. The reactions involved are described in Figure 22.

$$
\begin{aligned}
& \mathrm{U}_{1}+\mathrm{U}_{1} \underset{\gamma 20}{\stackrel{\kappa 11}{\rightleftharpoons}} \mathrm{U}_{2} \quad \mathrm{U}_{2}+\mathrm{U}_{2} \underset{\gamma 22}{\stackrel{\alpha \kappa 22}{\leftrightarrows}} \mathrm{U}_{2} \\
& \mathrm{U}_{1}+\mathrm{U}_{2} \stackrel{\mathrm{\kappa} 12}{\underset{\gamma 21}{\rightleftarrows}} \mathrm{U}_{2} \quad \mathrm{U}_{2}+\mathrm{U}_{2} \underset{\gamma 30}{\stackrel{\beta \kappa 22}{\rightleftharpoons}} \mathrm{U}_{3} \\
& \mathrm{U}_{1}+\mathrm{U}_{3} \underset{\gamma 31}{\stackrel{\kappa 13}{\leftarrow-1}} \mathrm{U}_{3} \quad \mathrm{U}_{2}+\mathrm{U}_{3} \frac{\kappa 23}{\gamma 32} \mathrm{U}_{3}
\end{aligned}
$$

Figure 22: The association and disassociation between the three species, namely, monomers $\left(U_{1}\right)$, oligomers $\left(U_{2}\right)$ and plaques $\left(U_{3}\right)$.

The left column includes reactions that describe monomers binding with monomers, oligomers and plaques to form oligomers and plaques; the right column includes those of the oligomers binding with themselves forming larger oligomers but some $(\alpha)$ proportion of these becoming plagues, and oligomers binding with plaques. The backward reaction is assumed for each forward reaction, although it is unlikely that plaques will break off into small fragments and thus $\gamma_{3, i} \approx 0$ is assumed for all $i$. Both monomers $U_{1}$ and oligomers $U_{2}$ are able to move around and can be cleared by ISF flow or through diffusion where as plaques $U_{3}$ are not and so the effect of advection and diffusion is assumed negligible and thus the governing equations are as follows,

$$
\begin{aligned}
\frac{\partial u_{1}}{\partial t}+\nabla \cdot\left(v u_{1}\right) & =D_{1} \nabla^{2} u_{1}+f_{\phi}-2 \kappa_{11} u_{1}^{2}-\kappa_{12} u_{1} u_{2}-\kappa_{13} u_{1} u_{p}+2 \gamma_{20} u_{2}+\gamma_{21} u_{2}+\gamma_{31} u_{3}-\delta_{1} u_{1} \\
\frac{\partial u_{2}}{\partial t}+\nabla \cdot\left(v u_{2}\right) & =D_{2} \nabla^{2} u_{2}+\kappa_{11} u_{1}^{2}-(1+\beta) \kappa_{22} u_{2}^{2}-\kappa_{23} u_{2} u_{3}-\gamma_{20} u_{2}+\gamma_{22} u_{2}+2 \gamma_{30} u_{3}+\gamma_{32} u_{3}-\delta_{2} u_{2} \\
\frac{\partial u_{3}}{\partial t} & =\beta \kappa_{22} u_{2}^{2}-\gamma_{30} u_{3}-\delta_{3} u_{3}
\end{aligned}
$$

where $v$ is the ISF velocity, $f_{\phi}$ is the constant production rate of $\mathrm{A} \beta$ monomers by cells and $\delta_{i}$ 's the degradation rate constants (we expect $\delta_{3} \leq \delta_{2} \leq \delta_{1}$ ). To understand better the important dynamics of the PDE system, we examine the spatially homogeneous case, namely,

$$
\begin{aligned}
\frac{d u_{1}}{d t} & =f_{\phi}-\left(\kappa_{11}+\bar{\kappa}_{1}\right) u_{1}^{2}-\kappa_{12} u_{1} u_{2}-\kappa_{13} u_{1} u_{3}+2 \gamma_{20} u_{2}+\gamma_{21} u_{2}-\delta_{1} u_{1} \\
\frac{d u_{2}}{d t} & =\bar{\kappa}_{1} u_{1}^{2}-\left(\kappa_{22}+\bar{\kappa}_{2}\right) u_{2}^{2}-\kappa_{23} u_{2} u_{3}-\gamma_{20} u_{2}+\gamma_{22} u_{2}-\delta_{2} u_{2} \\
\frac{d u_{3}}{d t} & =\bar{\kappa}_{2} u_{2}^{2}-\delta_{3} u_{3}
\end{aligned}
$$

assuming $\gamma_{3 i}=0, \forall i$ with $\bar{\kappa}_{1}=\kappa_{11}$ and $\bar{\kappa}_{2}=\beta \kappa_{22}$. The steady state analysis of this system is discussed in Section 3. 


\section{Appendix B: The immersed boundary method for 2D simula- tions}

The immersed boundary formulation of Equations (2.14)-(2.17) is solved in a square computational domain, $\Omega=\Omega^{+} \cup \Omega^{-}$, as shown in Figure 15, where the brain outer boundary with the subarachnoid and the inner ventricular boundary are embedded in a square box bounded by $\partial \Omega^{+}$, which is parameterised as a vector valued function of $\alpha$, namely $\mathbf{X}(\alpha)=(X(\alpha), Y(\alpha))$. The computational domain, $\Omega$, is further divided by the introduction of an indicator function $H$ which has the following property:

$$
H(x, t)= \begin{cases}0, & x \in \Omega^{-}, \\ 1, & x \in \Omega^{+} .\end{cases}
$$

Given that the material derivative of $H$ is zero, i.e. $\frac{D H}{D t}=0$, and $\nabla H=\int_{\partial \Omega^{+}} \boldsymbol{n} \delta(\boldsymbol{x}-\mathbf{X}) d \alpha$, as a result,

$$
\frac{\partial H}{\partial t}+\boldsymbol{v} \cdot \int_{\partial \Omega^{+}} \boldsymbol{n} \delta(\boldsymbol{x}-\mathbf{X}) d \alpha=0
$$

with $n$ being the unit outward normal vector on the interface pointing from $\Omega^{+}$into $\Omega^{-}$.

Next, we derive the unified equations valid in the entire domain $\Omega$ by means of the indicator function. Extending functions in $\Omega^{ \pm}$into the entire $\Omega$, the unified parameters, velocity field, $v$, pressure, $p$, and the concentrations of $\mathrm{A} \beta$ species can be expressed as

$$
\begin{array}{cl}
s=s^{+} H+s^{-}(1-H), & \boldsymbol{v}=\boldsymbol{v}^{+} H+\boldsymbol{v}^{-}(1-H), \\
p=p^{+} H+p^{-}(1-H), & u_{m}=u_{m}^{+} H+u_{m}^{-}(1-H),
\end{array}
$$

where $\alpha$ represents all scalar parameters. The product of the unified scalar parameter and the scalar function (or two scalar functions) is represented using the unified expression

$$
\begin{aligned}
s u_{m} & =\left(s^{+} H+s^{-}(1-H)\right)\left(u_{m}^{+} H+u_{m}^{-}(1-H)\right) \\
& =s^{+} u_{m}^{+} H+s^{-} u_{m}^{-}(1-H) .
\end{aligned}
$$

Similarly, the product of a unified scalar function and a vector valued function, $f$, is

$$
f u_{j}=f^{+} u_{j}^{+} H+f^{-} u_{j}^{-}(1-H) .
$$

The gradient of a unified scalar function, $\phi$, has the formula

$$
\begin{aligned}
\nabla \phi & =\nabla\left(\phi^{+} H+\phi^{-}(1-H)\right) \\
& =\nabla \phi^{+} H+\nabla \phi^{-}(1-H)+\int_{\partial \Omega^{+}}\left(\phi^{+}-\phi^{-}\right) \boldsymbol{n} \delta(\boldsymbol{x}-\mathbf{X}) d \alpha .
\end{aligned}
$$

Finally, the divergence of a unified vector valued function, $f$, is derived as follows:

$$
\nabla \cdot f=\left(\nabla \cdot f^{+}\right) H+\left(\nabla \cdot f^{-}\right)(1-H)+\int_{\partial \Omega^{+}}\left(f^{+}-f^{-}\right) \cdot \boldsymbol{n} \delta(\boldsymbol{x}-\mathbf{X}) d \alpha .
$$


The derivation of the unified concentration equations involves the following terms:

$$
\begin{aligned}
& \frac{\partial u_{m}}{\partial t}=\frac{\partial u_{m}^{+}}{\partial t} H+\frac{\partial u_{m}^{-}}{\partial t}(1-H)-\left(u_{m}^{+}-u_{m}^{-}\right) \boldsymbol{v} \cdot \int_{\partial \Omega^{+}} \boldsymbol{n} \delta(\boldsymbol{x}-\mathbf{X}) d \alpha \\
& \nabla \cdot\left(-\boldsymbol{v} u_{m}+\frac{D_{m}}{P e} \nabla u_{m}\right)= \nabla \cdot\left(\left(-\boldsymbol{v} u_{m}+\frac{D_{m}}{P e} \nabla u_{m}\right)^{+}\right) H \\
&+\nabla \cdot\left(\left(-\boldsymbol{v} u_{m}+\frac{D_{m}}{P e} \nabla u_{m}\right)^{-}\right)(1-H) \\
&+\int_{\partial \Omega^{+}}\left[-\boldsymbol{v} u_{m}+\frac{D_{m}}{P e} \nabla u_{m}\right]_{\partial \Omega^{+}} \cdot \boldsymbol{n} \delta(\boldsymbol{x}-\mathbf{X}) d \alpha,
\end{aligned}
$$

and

$$
f_{m}=f_{m}^{+} H+f_{m}^{-}(1-H),
$$

where $\left[-v u_{m}+\frac{D_{m}}{P e} \nabla u_{m}\right]_{\partial \Omega^{+}}=\left(-v u_{m}+\frac{D_{m}}{P e} \nabla u_{m}\right)^{+}-\left(-v u_{m}+\frac{D_{m}}{P e} \nabla u_{m}\right)^{-}=Q_{m} u_{m}$ is the jump across the interface. The continuity of concentration and boundary conditions give

$$
\begin{aligned}
\frac{\partial u_{m}}{\partial t}+\nabla \cdot\left(v u_{m}\right) & =\frac{D_{m}}{P e} \nabla^{2} u_{m}+f_{m}-\int_{\partial \Omega^{+}} Q_{m} u_{m} \delta(x-\mathbf{X}) d \alpha, \\
\frac{d u_{M}}{d t} & =f_{M} .
\end{aligned}
$$

Similarly, the unified governing equation of pressure is

$$
-\nabla^{2} p=\frac{1}{K} S(\phi)+\int_{\partial \Omega^{+}} \eta(\alpha) \delta(x-\mathbf{X}) d \alpha,
$$

where $\eta(\alpha)$ is the normal jump condition of pressure gradient derived from the integration of Equation (2.14).

\section{B.1 Numerical schemes}

The immersed boundary method $[26,27,28]$ is a hybrid method using the Eulerian $(x=(x, y))$ and Lagrangian $(\mathbf{X}=(X(\alpha), Y(\alpha)))$ representations. Here, the computational domain $\Omega=$ $[a, b] \times[c, d]$, and $\partial \Omega^{+}$represents the boundary of subarachnoid and ventricular boundaries which are embedded interfaces in $\Omega$. In the fixed coordinates, fluid variables are defined on a staggered marker-and-cell (MAC) mesh introduced by Harlow and Welch [?]. The vectorvalued concentration function $u$ is a column vector including $u_{m}, m=1, \ldots, 5$ as entries are defined on the grid points labelled as $\left(x_{i}, y_{j}\right)=(a+(i-1 / 2) h, c+(j-1 / 2) h)$ for $i, j=1,2 \ldots, N$. The velocity components $v^{x}$ and $v^{y}$ are defined at $\left(x_{i+1 / 2}, y_{j}\right)=(a+i h, c+(j-1 / 2) h)$ and $\left(x_{i}, y_{j+1 / 2}\right)=(a+(i-1 / 2) h, c+j h)$, respectively, where $h=\Delta x=\Delta y$. In addition, the pressure is defined at the same location as the concentration. For each immersed interface, we choose a fixed parameter $\alpha \in[0,1]$ for convenience, and use a collection of discrete points $\alpha_{k}=k \Delta \alpha, k=0,1, \ldots L$ such that the Lagrangian immersed boundary points are denoted by $\boldsymbol{X}_{k}=\boldsymbol{X}\left(\alpha_{k}\right)=\left(X_{k}, Y_{k}\right)$, where $\Delta \alpha=1 / L$.

Let $\Delta t$ be the time step size and $n$ be the time step index. At the beginning of each time step, the variables $\boldsymbol{X}_{k}^{n}=\boldsymbol{X}\left(\alpha_{k}, n \Delta t\right), \boldsymbol{u}^{n}=\boldsymbol{u}(\boldsymbol{x}, n \Delta t)$ and $\boldsymbol{v}^{n}=\boldsymbol{v}(\boldsymbol{x}, n \Delta t)$ are given. The variables at the next time step $(n+1)$ are obtained via numerical integration in the following steps. 
1. Computation of indicator function $H$ and velocity fields $v$ : solve Eq. (B.9) for pressure by second-order accurate Poisson solver, and calculate $v$ by centred difference scheme. Since we assume the embedded interfaces are fixed and the porosity $\phi$ is not affected by $u$, the pressure $p$ and velocity $v$ are not changed in the numerical integration.

2. Interpolate the current $u^{n}$ from the fixed grid points onto the immersed boundary points to obtain interfacial concentration $\boldsymbol{U}_{k}^{n}$.

$$
\boldsymbol{U}_{k}^{n}=\sum_{\boldsymbol{x}_{i, j}} \boldsymbol{u}_{i, j}^{n} \delta_{h}\left(x_{i}-X_{k}^{n}\right) \delta_{h}\left(y_{j}-Y_{k}^{n}\right) h^{2}, \quad k=0,1, \ldots L,
$$

where a smooth delta function is given by

$$
\delta_{h}(r)= \begin{cases}\frac{1}{8 h}\left(3-2 \frac{|r|}{h}+\sqrt{1+4 \frac{|r|}{h}-4 \frac{|r|^{2}}{h^{2}}}\right), & \text { if } 0 \leq|r| \leq h, \\ \frac{1}{8 h}\left(5-2 \frac{|r|}{h}-\sqrt{\left.-7+12 \frac{|r|}{h}-4 \frac{|r|^{2}}{h^{2}}\right),},\right. & \text { if } h \leq|r| \leq 2 h, \\ 0, & \text { otherwise. }\end{cases}
$$

3. Calculate the boundary flux condition $G$ and spread interfacial sources from the immersed boundary points to the Eulerian grid by

$$
\begin{aligned}
\boldsymbol{G}_{k}^{n} & =Q \boldsymbol{U}_{k^{\prime}}^{n} \\
\bar{u}^{n}\left(\boldsymbol{x}_{i, j}\right) & =\sum_{k=1}^{L}(G)_{k}^{n} \delta_{h}\left(x_{i}-X_{k}^{n}\right) \delta_{h}\left(y_{j}-Y_{k}^{n}\right) \Delta \alpha,
\end{aligned}
$$

where $\bar{u}_{m}$ is the source term from the boundary condition.

4. Solve the concentration equations: the time derivative is approximated by a first-order backward difference formula and the nonlinear term is approximated by extrapolation using the values from the previous time steps. For $m=1, \ldots, M-1$,

$$
\frac{u_{m}^{n+1}-u_{m}^{n}}{\Delta t}+\left(\nabla_{h}\right) \cdot\left(v u_{m}^{n}\right)=\frac{D_{m}}{P e} \Delta_{h} u_{m}^{n+1}+f_{m}^{n}-\bar{u}_{m}^{n}, \quad \text { for } \quad m=1, \ldots, M-1,
$$

and the plaque is updated by

$$
\frac{u_{M}^{n+1}-u_{M}^{n}}{\Delta t}=\bar{k}_{M-1, M-1}\left(u_{M-1}^{2}\right)^{n}-r_{M, 0} u_{M}^{n}-\delta_{M} u_{M}^{n+1}
$$

Here, $\nabla_{h}$ and $\Delta_{h}$ are discrete divergence and Laplace operators defined on staggered grid. Notice that we have used centred-difference numerical scheme for advection term due to the small Peclet number we considered in all numerical simulations. An upwind type numerical scheme should be included when the system is advection dominated. 